\title{
Extracting interstellar diffuse absorption bands from cool star spectra
}

\section{Application to bulge clump giants in Baade's window}

\author{
H.-C. Chen ${ }^{1}$, R. Lallement ${ }^{1}$, C. Babusiaux ${ }^{1}$, L. Puspitarini ${ }^{1}$, P. Bonifacio ${ }^{1}$, and V. Hill ${ }^{2}$ \\ ${ }^{1}$ GEPI Observatoire de Paris, CNRS, Université Paris-Diderot, Place Jules Janssen, 92195 Meudon Cedex, France \\ e-mail: hui-chen. chen@obspm. fr \\ 2 Université de Nice Sophia Antipolis, CNRS, Observatoire de la Côte d'Azur, Laboratoire Cassiopée, BP 4229, \\ 06304 Nice Cedex 4, France
}

Received 19 September 2012 / Accepted 5 November 2012

\section{ABSTRACT}

\begin{abstract}
Aims. Interstellar (IS) absorption lines or diffuse bands are usually extracted from early-type star spectra because they are characterized by smooth continua. However, this introduces a strong limitation on the number of available targets, and reduces potential studies of the IS matter and the use of absorptions for cloud mapping.

Methods. We have developed a new and automated fitting method appropriate to interstellar (IS) absorptions in the spectra of cool stars that possess stellar atmospheric parameters. We applied this method to the extraction of three diffuse interstellar bands (DIBs) in high resolution VLT FLAMES/GIRAFFE spectra of red-clump stars from the bulge. By combining all stellar synthetic spectra, HITRAN-LBLRTM atmospheric transmission spectra and diffuse band empirical absorption profiles, we determined the 6196.0, 6204.5, and 6283.8 $\AA$ DIB strength toward the 219 target stars and discuss the sources of uncertainties. To test the sensitivity of the DIB extraction, we compared all three results and compared the DIB equivalent widths (EWs) with the reddening derived from an independent extinction map based on OGLE photometric data. We also derived the three independent color excess estimates based on the DIB strengths and average linear correlation coefficients previously established in the solar neighborhood and compared with the OGLE photometric results.

Results. Most stellar spectra could be reproduced well by the composite stellar, atmospheric, and IS models. Measurement uncertainties on the EWs are smaller for the broad and strong $6283.8 \AA \mathrm{DIB}$, and are in the range 10-15\%. Uncertainties on the two narrow and weaker DIBs are larger, as expected, and found to be highly variable from one target to the next. They strongly depend on the star's radial velocity. DIB-DIB correlations among the three bands demonstrate that a meaningful signal is extracted. For the 6284 and $6204 \AA$ DIBs, the star-to-star variability of the EW also reflects features of the OGLE extinction map. The three independent extinction estimates deduced from the EWs and solar neighborhood correlation coefficients agree with each other within $20 \%$, a value which probably reflects that they belong to different families. The estimated average color excess is also compatible with the photometric determination.

Conclusions. This work demonstrates the feasibility of the method of ISM DIB extraction in cool star spectra, based on synthetic spectra. It confirms that DIB measurements and local DIB-extinction calibrations can provide rough, first-order estimates of the towards distant targets.
\end{abstract}

Key words. ISM: lines and bands - dust, extinction - ISM: structure - ISM: molecules - stars: late-type - methods: data analysis

\section{Introduction}

Diffuse interstellar bands (DIBs) and interstellar (IS) absorption lines have been almost exclusively extracted from early-type star spectra, using the fact that stellar lines are broad, shallow, and limited in number, and that they do not contaminate the IS lines. The stellar continua can be easily fitted without any need for stellar models. However, extracting absorption from early-type star spectra is a strong limiting factor since it considerably reduces the number of potential targets, the information that can be obtained on the source regions of the absorptions, and especially the spatial resolution that can be achieved. In the case of the DIBs, it limits studies of the DIB response to the radiation field and to the local physical properties within the IS clouds, while such studies are promising tools in the search for the DIB carriers (e.g., Vos et al. 2011). Moreover, with the advent of stellar spectroscopic surveys at increasing resolution, the use of multi-object spectrographs and the perspective of forthcoming Gaia parallaxes, a large amount of line-of-sight integrated absorption data will become available.

Extracting absorption characteristics towards all targets, including cool stars, would allow better mapping of the galactic ISM, because it would considerably increase the achievable spatial resolution. For such mapping, IS lines of atoms, simple molecules, and DIBs are available, since all three contain information on the amount of IS matter. While the first two are well identified, carriers of the last remain unknown, despite their early discovery (e.g., Herbig 1995; Jenniskens \& Desert 1994; Salama et al. 1996; Fulara \& Krelowski 2000; Snow \& Destree 2011; Friedman et al. 2011; Hobbs et al. 2008, 2009). Still, this lack of carrier identification and the fact that most of them are only weakly correlated with extinction or gas column are not incompatible with their use as tracers of IS clouds in the same way IS lines are used; i.e., the radial gradients of their strengths allow 
locating IS clouds in distance and building 3D maps. In some respects, DIBs may have advantages as interstellar medium (ISM) tracers, because they are numerous, thus observable at various instrumental settings; they are often broad; and in general they are unsaturated. Recent data analyses suggest that the average ratios between the DIBs and the extinction do not seem to vary significantly within the first kiloparsec (Friedman et al. 2011; Vos et al. 2011). Finally, making use of cooler stars to extract their strengths reduces the dispersion around the average relationship, because extreme ionizing field effects linked to bright and hot targets are avoided (Raimond et al. 2012). For this reason, extracting DIB information from cool stars would be another improvement to the mapping.

Here we present a new method of DIB measurement based on cool star synthetic spectra, and as a test case we applied this technique to three different diffuse bands and 219 targets located in the Galactic bulge that have the advantage of possessing precise determinations of their atmospheric parameters. We selected the 6196.0, 6204.5, and 6283.8 $\AA$ DIBs that are the three strongest absorptions contained in the observed spectral interval. Those DIBs have already been widely studied (see e.g., the works of Galazutdinov et al. 2008; Cami et al. 1997; Friedman et al. 2011; Vos et al. 2011). We estimate the statistical and systematic uncertainties on the DIB equivalent widths (EWs) and discuss potential improvements. We compare the measured EW angular pattern over the field with an independent mapping of the reddening based on photometric data of stars close to the Galactic center (GC). We finally use average DIB-reddening empirical relationships previously established in the Sun's vicinity to derive three independent estimates of the reddening towards all targets, compare the three estimate together, and finally compare with the photometric determination.

This article is organized as follows. Data are presented in Sect. 2. In Sect. 3 we describe the new method of IS absorption band extraction and its ingredients. In Sect. 4, we show the model adjustments for selected target stars and discuss the results. In Sect. 5, we discuss the relations between the DIBs and between the DIBs and the extinction obtained by photometry. Section 6 presents color excess estimates based on the DIBs and nearby star studies. Finally, we summarize the results and discuss the perspectives in the last section.

\section{Data}

We used the sample of 219 bulge red clump giants of Hill et al. (2011). Those stars were observed with FLAMES/GIRAFFE at the VLT and are located within a 12 arcmin radius field in Baade's Window $(l=0.8, b=-4)$. We used here the spectra obtained with the GIRAFFE HR13 setup, leading to a resolution of $R=22500$ and a spectral coverage spanning 6120 to $6405 \AA$, which contains three well known diffuse bands. The target selection, observations, data reduction, and stellar-parameter determination are described by Hill et al. (2011). Those stellar parameters were determined by these authors from photometric and spectroscopic data. Here we use the effective temperature, gravity, micro-turbulence, and metallicity resulting from their analysis, whose full ranges for the present sample are listed in Table 1. The different exposures were observed within a week interval so that at this resolution we can neglect variations in the radial velocity difference between the telluric and stellar lines and work with the final co-added spectra. Signal-to-noise ratios vary between $\simeq 30$ and 77 per pixel. (The Giraffe pixel-size is $0.07 \AA$.)
Table 1. Stellar parameters.

\begin{tabular}{lccc}
\hline \hline Parameters & Min & Max & Unit \\
\hline$T_{\text {eff }}$ & 4270 & 5448 & $\mathrm{~K}$ \\
$\log g$ & 1.97 & 2.46 & \\
$v_{\mathrm{t}}$ & 0.8 & 1.8 & $\mathrm{~km} \mathrm{~s}^{-1}$ \\
{$[\mathrm{Fe} / \mathrm{H}]$} & -1.13 & 0.71 & \\
\hline
\end{tabular}

Notes. $T_{\text {eff }}$ is the effective temperature. $\log g$ is the gravity. $v_{\mathrm{t}}$ is the micro-turbulent velocity. $[\mathrm{Fe} / \mathrm{H}]$ is the metallicity.

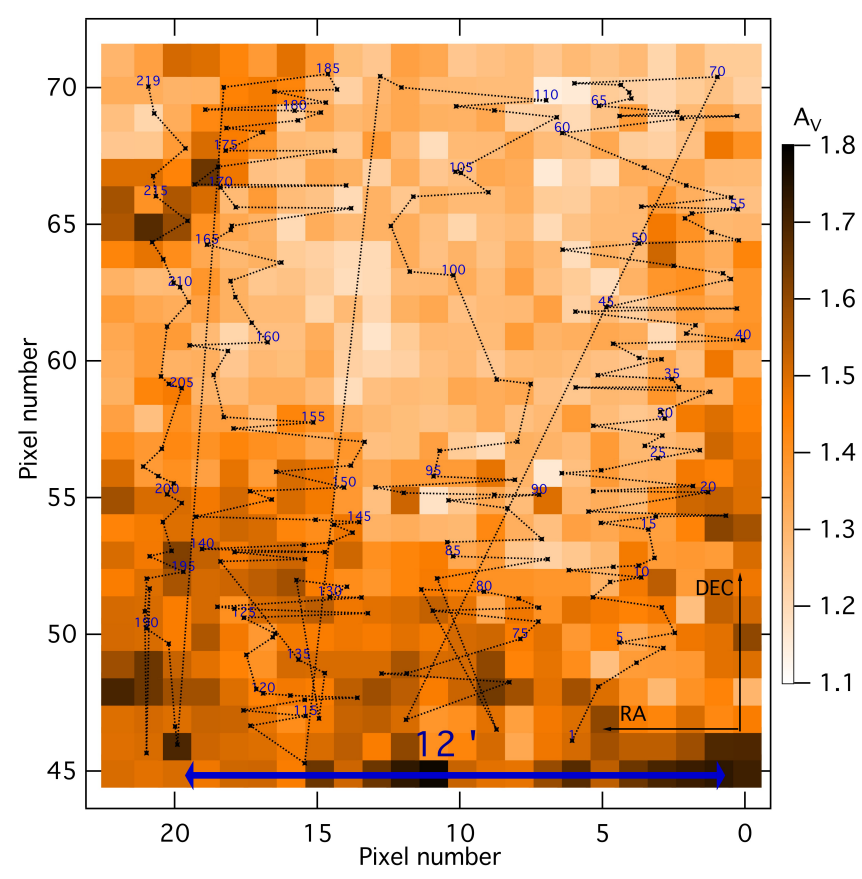

Fig. 1. Target stars used in this study (black crosses) superimposed on the extinction map from Sumi (2004). The map is centered on $(l, b)=$ $(0.8,-4)$. Each pixel is $0.6^{\prime} \times 0.6^{\prime}$. The extinction scale is displayed in Fig. 14. Stars are numbered according to Table 3. Dotted lines link stars in increasing order of their ID number. Only one out of five numbers is indicated for clarity.

This region benefits from a high-resolution differential extinction map that was derived from the OGLE-II red clump giants photometry by Sumi (2004). Assuming a mean reddeningcorrected color for the red clump, Sumi (2004) divided each field in small subfields, computed the mean observed color of the red clump in each subfield and derived the reddening $E_{V-I}$. The resulting extinction map has a spatial resolution of 33 arcsec in our field. For comparison purposes we have interpolated within the Sumi (2004) reddening grid to infer its value at each target location. Sumi (2004) indicates a potential zero-point offset for $A_{v}$ that may amount to 0.05 . The distribution of the target stars is shown superimposed on the Sumi (2004) extinction map in Fig. 1.

\section{Fitting procedure}

Our approach is to model and adjust the spectrum of the background star, the DIB, and the atmospheric transmission simultaneously in one unique step. In the opposite case of hot stars, extracting the IS absorption was done in two or three steps. The correction for the telluric absorption, if necessary, was done first and independently of the IS absorption fitting. Synthetic 
transmission spectra were adjusted through computing the mean ratio between observed and modeled EWs of specific lines (Lallement et al. 1993), or by minimizing the length (defined as the sum of flux differences between consecutive points) of the residual spectrum obtained after dividing by the model atmospheric transmission (Raimond et al. 2012). None of those methods is necessary here, since the atmospheric transmission model is adjusted in velocity and airmass during the global adjustment. In a second step the smooth continuum of the hot star was fitted to a function, and in a last step the DIB strength was measured using the normalized spectrum. Characterizing the DIB strength was done in several ways, the two main ones being measurements of the central depth and of the EW. We refer to the detailed discussion of this point by Friedman et al. (2011). We chose here the EW, and, as we will see, this is the quantity that comes directly out from our cool star analysis.

For cool stars, the presence of numerous, deep, and narrow stellar lines precludes applying the simple continuum fitting procedure that is used for stars of earlier spectral types. Identifying and correcting the stellar lines one by one in the IS absorption region is not practical. Furthermore, if the stellar lines and the IS absorption do overlap, it is very difficult or even impossible to separate them, hence our approach using a global adjustment. Our composite model here is the product of a synthetic stellar spectrum, a synthetic telluric transmission, and an empirical model for the DIB absorption. The model allows for wavelength shifts between those three spectra to take the stellar radial velocity, the Earth motion, and the IS radial velocity into account. The giant stars used as background sources in the present investigation do not have measurable projected rotational velocities at this resolution. Sizeable projected rotational velocity in the background star could be modeled as well, if necessary. The combination of the three models is convolved by a Gaussian profile to take the instrumental spectral resolution into account and is adjusted to the data in the DIB region, with the DIB strength, the DIB center location, and an atmospheric transmission scaling (see below) as the free parameters.

The synthetic stellar spectra, $S(\lambda)$, were computed from an ATLAS 9 model atmosphere using the SYNTHE suite (Kurucz 2005). We used the Linux port of all the codes (Sbordone et al. 2004; Sbordone 2005). Atomic and molecular data were taken from the data base on Kurucz's website ${ }^{1}$ (Kurucz 2005). For each star we computed a synthetic spectrum with the stellar parameters obtained by Hill et al. (2011), i.e. the effective temperatures, gravity, metallicity, and microturbulence, summarized in Table 1. The stellar radial velocity is taken into account by Doppler-shifting the computed spectrum to the appropriate radial velocity.

The synthetic telluric transmissions were computed by means of the LBLRTM code (Line-By-Line Radiative Transfer Model, Clough et al. 2005), using the molecular database HITRAN (HIgh-resolution TRANsmission molecular absorption, Rothman et al. 2009). Here we have used the transmission $T_{0}(\lambda)$ computed for a standard atmosphere and for the altitude of the observatory and assumed that airmass variations from star to star simply result in a variable coefficient $\alpha$ for the transmission, with $T(\lambda)=T_{0}(\lambda)^{\alpha}$. The projected Earth motion is taken into account by Doppler-shifting the transmission.

Finally, the profiles of the $6196.0,6204.5$, and $6283.8 \AA$ DIBs are derived from a high resolution $(R=48000)$, high signal-to-noise survey of early-type stars recorded with the FEROS spectrograph at the $2.2 \mathrm{~m} \mathrm{ESO/Max} \mathrm{Planck} \mathrm{Institute}$

http://kurucz .harvard.edu
Telescope in La Silla. Those profiles do not reveal the fine structure that is known to be present Galazutdinov et al. (2008), but this is not necessary here since we deal with spectra at a resolution that is lower than the one of FEROS. The shape of the 6283.8 $\AA$ DIB, which is contaminated by strong telluric molecular oxygen lines, has been derived by Raimond et al. (2012) by averaging a large number of individual shapes obtained after division by an adjusted telluric template. This use of a synthetic atmospheric model is allowed by this DIB being relatively strong and much broader than the telluric lines. In the same way, this DIB is broader here than the stellar lines and thus relatively easy to detect and measure (see Figs. 3-5). The two, much weaker DIBs, 6196.0 and $6204.5 \AA$, are in regions that are free of contaminating telluric lines. Their shapes were derived by means of an automated fitting method appropriate to early-type stars (Puspitarini et al. 2012, priv. comm.). About ten individual profiles extracted from the best FEROS spectra were averaged to produce those templates. Since those two DIBs are narrower than the 6283.8 $\AA$ DIB, here in the case of cool stars they are much more difficult to measure due to the overlap with the similarly narrow stellar lines.

Neglecting emissions from the cloud in comparison with the stellar flux, DIB transmission profiles $D(\lambda)$ can be expressed as $\exp (-\tau(\lambda))$, where $\tau(\lambda)$ is the optical thickness as a function of wavelength. The value of $\tau(\lambda)$ is proportional to the column $N$ of absorbers and can be expressed as $\tau(\lambda)=N / N_{0} \tau_{0}(\lambda), \tau_{0}(\lambda)$ and $N_{0}$ being some related optical thickness and column of reference. Calling $\beta$ the $N / N_{0}$ ratio, a quantity proportional to the column density of the DIB carrier, one has $D(\lambda)=D_{0}\left(\lambda_{\mathrm{D}}\right)^{\beta}$, where $D_{0}(\lambda)=\exp \left(-\tau_{0}(\lambda)\right)$ is the reference profile derived from the FEROS analyses, and $\lambda_{\mathrm{D}}$ is the wavelength shifted by the radial velocity of the main absorbing medium. The DIB EW is, by definition,

$W=\int \frac{I_{0}(\lambda)-I(\lambda)}{I_{0}(\lambda)} \mathrm{d} \lambda=\int(1-\exp (-\tau(\lambda))) \mathrm{d} \lambda$

where $I_{0}(\lambda)$ and $I(\lambda)$ are the unabsorbed and absorbed intensities. Within the weak absorption regime appropriate here, $\tau$ is small, $\exp (-\tau) \simeq 1-\tau$ and the EW $W$ is approximated by

$W=\int \tau(\lambda) \mathrm{d} \lambda=\int \beta \tau_{0}(\lambda) \mathrm{d} \lambda=\beta W_{0}$

where $W_{0}$ is the EW of the line of reference.

We chose to list the results in the form of the product $\beta W_{0}$, which has the advantage of being both truly proportional to the absorbing column and being an equivalent width, i.e., a widely used, meaningful quantity. For the broader DIB, $6284 \AA$ A, the relative difference between $\beta W_{0}$ and the actual EW is smaller here than $4.5 \%$, a value reached for EWs on the order of $1.2 \AA$. For the narrower DIB, $6196 \AA$, the departure from the linear regime for the EW occurs at a smaller optical thickness; however, the DIB is weak, and finally the relative difference between $\beta W_{0}$ and the actual EW is less than $4 \%$, a value reached for EWs on the order of $70 \mathrm{~m} \AA$.

After adjusting the coefficient $\beta$ through spectral fitting, the DIB EW is thus simply computed as the product of the EW of the reference profile $W_{0}$ by the coefficient $\beta$. We also assume here that most of the absorbing medium originates in clouds with a similar radial motion, or equivalently that the DIB is negligibly broadened by the line-of-sight velocity structure. This assumption is legitimated here by the fact that most of the absorption arises within $1500 \mathrm{pc}$ from the Sun, as derived from the extinction model of Marshall et al. (2006) and that for those low 1, b 


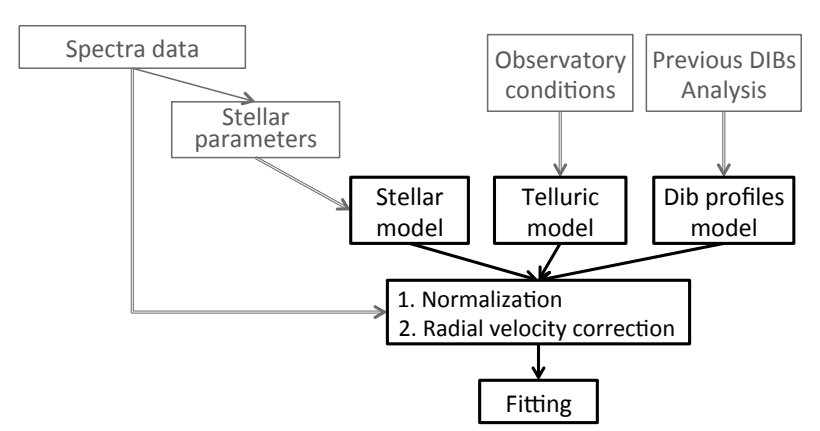

Fig. 2. Procedure flowchart of the fitting.

coordinates the projection of the gas motions onto the line-ofsight remains small with respect to the DIB spectral width.

Figure 2 illustrates the procedure for this study. The stellar parameters are used as input for the stellar synthetic models, and the stellar radial velocity is taken into account by an appropriate shift. The atmospheric synthetic model of reference is calculated based on the position and altitude of the observatory. While doing the fitting, the program computes the product $\kappa S\left(\lambda_{S}\right) T_{0}\left(\lambda_{E}\right)^{\alpha} D_{0}\left(\lambda_{\mathrm{D}}\right)^{\beta}$, with $\kappa$ being a scaling factor, $\lambda_{S}$ is the wavelength shifted by the stellar radial velocity, $\lambda_{E}$ by the Earth's radial velocity and $\lambda_{D}$ by the radial velocity of the DIB absorbing medium, and convolves the product of the three models by a Gaussian instrumental function appropriate for the resolution of GIRAFFE.

Telluric absorptions should be identical for all fibers for the same exposure, and we also expect the DIB radial velocity to vary very little over the narrow field. Still, to test the adjustment and allow for small uncertainties in the three wavelength shifts, we started by allowing all parameters $\kappa, \alpha, \beta, \lambda_{\mathrm{S}}, \lambda_{\mathrm{E}}$ and $\lambda_{\mathrm{D}}$ to freely vary, and performed a least mean squares adjustment to the data for each star. All the fittings were done in an automated way. The results revealed a large majority of identical or very close values for the $\lambda_{\mathrm{E}}$ shift (actually a null value for a fit in the earth frame) and for $\alpha$. As mentioned previously this was expected for both $\alpha$, which responds to the airmass, and $\lambda_{\mathrm{E}}$, a function of the date. We extracted those two parameters and kept them fixed for the second adjustment phase. From this first attempt we could also check that the values found by fitting for $\lambda_{\mathrm{S}}$ differ from the radial velocities from Hill et al. (2011) by very small velocity intervals (on the order of $1 \mathrm{~km} \mathrm{~s}^{-1}$ ). We kept those very small differences from values in Table 3 and fixed $\lambda_{\mathrm{S}}$ for the next step. Finally, the coefficient $\lambda_{\mathrm{D}}$ that represents the DIB shift was also found to be the same or nearly the same for a large majority of the stars. This confirms that the DIB radial velocity variation is very small over such a small field of view, both because most of the absorption originates in the first two kpc, and also because the radial motion of the absorbing gas in this direction is small. We then fixed this shift $\lambda_{\mathrm{D}}$ at the most frequently found value. For all those four parameters we checked that the outliers correspond to low signal-to-noise spectra or to the presence of a spurious feature. We then proceeded to a second fit of all spectra, this time only for free $\kappa$ and $\beta$ coefficients.

\section{Model adjustments}

Figures 3-9 illustrate exemplary cases of model adjustments for the three 6196.0, 6204.5, and 6283.8 $\AA$ DIBs chosen for this study.
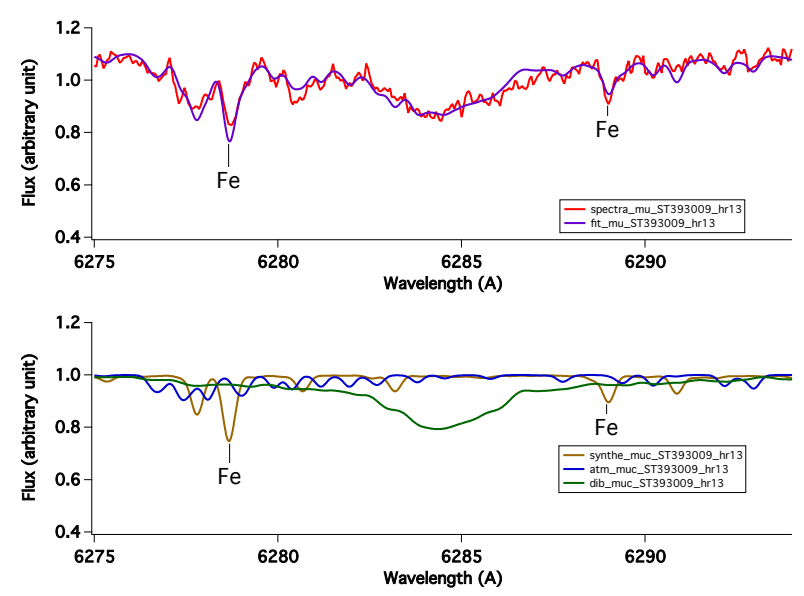

Fig. 3. Model adjustment for Ogle N: $393009\left(T_{\text {eff }}=5012 \mathrm{~K}\right)$. The DIB is in a region devoid of strong stellar lines. The upper panel shows the spectrum (red line) and the best-fit model (purple line). The lower panel shows the synthetic stellar model (yellow line), the synthetic atmospheric model (blue line), and the DIB profile (green line), which all correspond to the fit parameters.
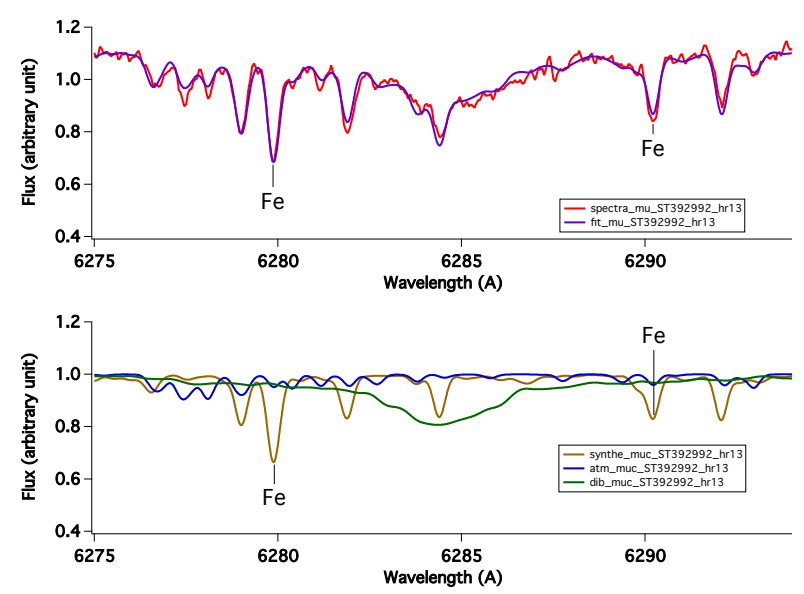

Fig. 4. Same as Fig. 3 for Ogle N: $392992\left(T_{\text {eff }}=4907\right.$ K). The DIB here is in a spectral region characterized by moderately strong stellar lines.
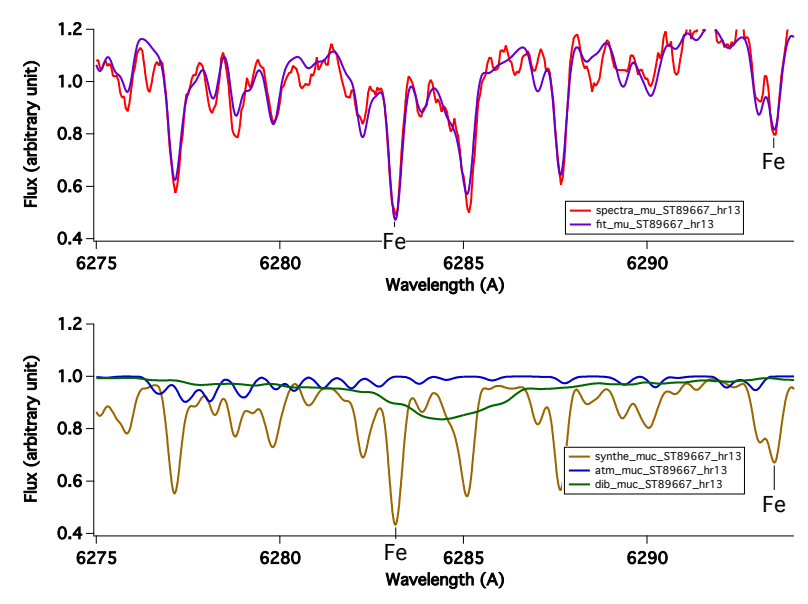

Fig. 5. Same as Fig. 3 for Ogle N: $89667\left(T_{\text {eff }}=4516 \mathrm{~K}\right)$. The DIB here is both weak and embedded in strong stellar lines. 
What we aim at showing in those figures are the strong differences linked to the target star individual radial velocities, which vary over a very large velocity interval, from -230 to $+250 \mathrm{~km} \mathrm{~s}^{-1}$. This results in very different locations of the DIBs with respect to the main stellar lines. There are also very large differences between the $6283.8 \AA$ DIB and the two narrow ones, with a much stronger impact of the star radial velocity and of the signal-to-noise on the DIB detection for the last two DIBs. Finally, the higher the metallicity, the stronger the stellar lines and their impact on the DIB measurement, especially again in the case of the two narrow and weak DIBs.

Figures 3 to 5 show three examples of adjustments for the strongest and broadest DIB at $6283.8 \AA$, The first star (Fig. 3) corresponds to an optimal case: given the star radial velocity, only weak stellar lines are located in the DIB region, and the fitting program could easily adjust the DIB strength. Moreover, the star is metal-poor $(\mathrm{Fe} / \mathrm{H}=-0.68)$, which helps the fitting procedure further. Figures 4 and 5 correspond to more difficult cases, with one or more strong stellar lines in the DIB region. In those cases, it should be possible to measure the DIB strengths with accuracy, provided the stellar models adequately predict the stellar line depths. However, it can be seen that there are significant and often systematic differences between the observed and modeled lines for some spectral regions. Such departures may have several origins: the oscillator strengths $(\log (g f))$ and other atomic data (wavelengths, damping constants) that we use have an error attached to them; some of the lines are simply not identified and absent from our line list; our spectra are computed assuming local thermodynamic equilibrium (LTE); departures from LTE in the stellar atmosphere may change the line strength, for a given abundance; the model atmospheres we used are one-dimensional static and plane-parallel, hydrodynamic effects (granulation) may also affect both the line strengths and shapes. In practice our computed stellar spectrum differs from the observed spectrum due to a number of shortcomings in our modeling. For example, significant residuals are found at the locations of unidentified lines of the solar spectrum, such as the 6273.949, 6282.816, 6286.142, and 6288.315 $\AA$ lines (Moore et al. 1966). Obviously such unidentified lines are not present in our line list. Details on these discrepancies can be found in the Appendix, which aims at estimating the uncertainties on the DIBs EWs and at empirically correcting for systematic effects. Nevertheless, despite the observed departures from the stellar model, the DIB is broad enough here for a reliable DIB estimate. Figure 5 illustrates this property, and shows one of the worst cases of overlapping stellar lines, a metal-rich (although moderately, $\mathrm{Fe} / \mathrm{H}=0.67$ ) target star, and one of the smallest DIB EWs. The DIB strength could be measured reasonably well despite those conditions, thanks to the good signal-to-noise ratio of the spectrum and the width of the DIB.

This is not the case for the two narrow DIBs. Figures 6 to 9 show two examples of determinations for each of the 6196.0 and 6204.0 ̊ DIBs, a relatively easy one with only weak stellar lines contaminating the DIB region, and a difficult one with stellar lines overlapping the DIB. In the first case, it can be seen that DIB EWs can be safely measured. In the second, it is clear from the figure that the stellar line accuracy is critical and that some of the measurements are very uncertain, at least for the low extinction (and DIB strength) regime that prevails here. In comparison with those uncertainties, additional errors linked to the use of a predefined shape of the DIB, as well as to the telluric model (for the $6284 \AA$ DIB), are negligible. Those systematic departures have been studied in detail and are discussed in the Appendix.
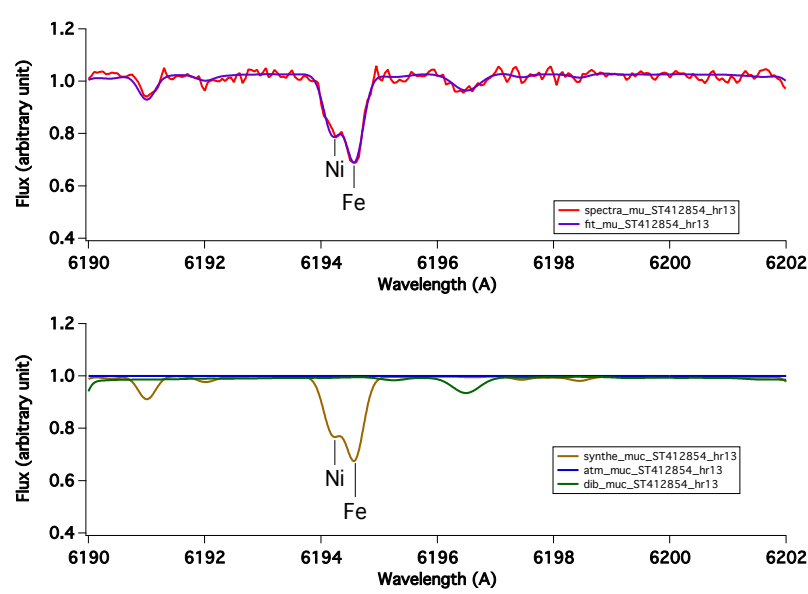

Fig. 6. Same as Fig. 3 for the $6196 \AA$ DIB and Ogle N: $412854\left(T_{\text {eff }}=\right.$ $5191 \mathrm{~K})$. The narrow DIB is in a region devoid of strong stellar lines.
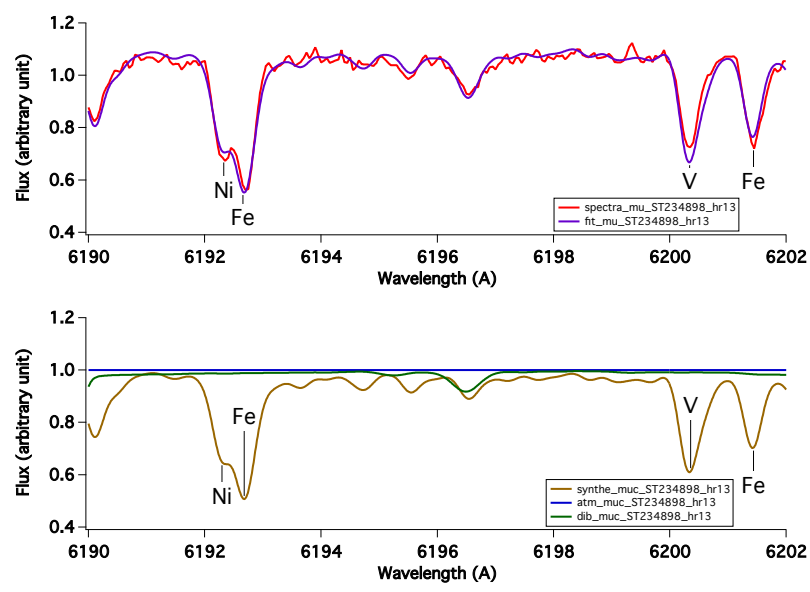

Fig. 7. Same a Fig. 6 for Ogle N: $234898\left(T_{\text {eff }}=4714 \mathrm{~K}\right)$. The DIB and a strong stellar line do overlap.

Based on them, a first-order empirical correction was devised and was applied to the DIB derivation. In brief, residuals for all spectra were all shifted to the stellar frame, and the resulting spectra were sorted as a function of the stellar metallicity (see Fig. A.1 in the Appendix). At each wavelength an average linear relationship between the residual value and the metallicity was adjusted, providing a systematic offset as a function of metallicity and wavelength (Figs. A.2 and A.3). This offset is maximal at the locations of the over- or underpredicted lines, and nil elsewhere. Such an offset was then applied as a corrective term at all wavelengths to all DIB spectra (Fig. A.4), and a new adjustment of the model and subsequent computation of the DIB EW were performed after those corrections. We compared the DIBDIB relationships and also the DIB-extinction relationships both before and after the empirical correction, and found a systematically better DIB-DIB relationship, in particular with a factor of two increase in the Pearson correlation coefficient in the case of the $6196 \AA$ vs. $6204 \AA$ DIB comparison. We also found a significant improvement in the DIB-extinction relationship, except for the $6284 \AA$ DIB for which there was no change. We are conscious that a more fundamental approach would be desirable, but, in view of those improvements, we kept the corrected values for the remaining part of the analysis. More improvements 

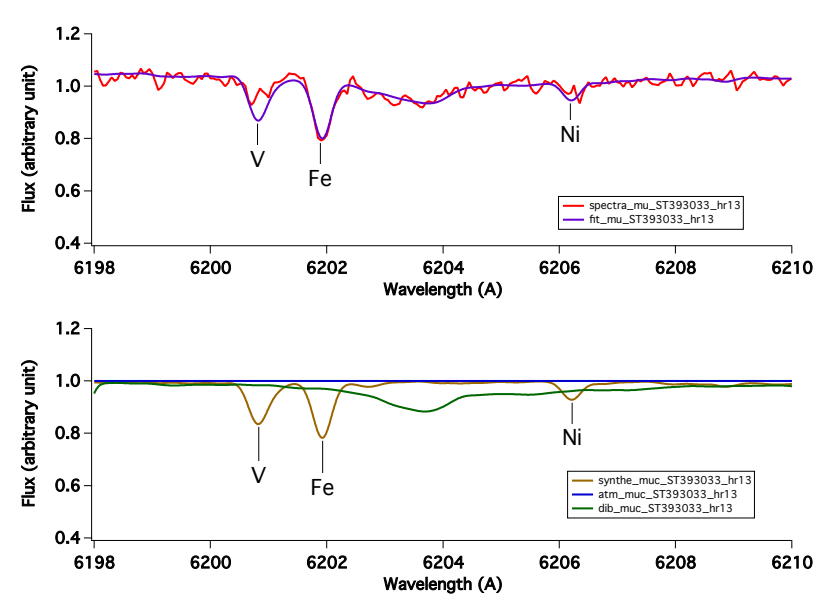

Fig. 8. Same as Fig. 3 for the $6204 \AA$ DIB and Ogle N: $393033\left(T_{\text {eff }}=\right.$ $4914 \mathrm{~K})$. The narrow DIB is in a region devoid of strong stellar lines.
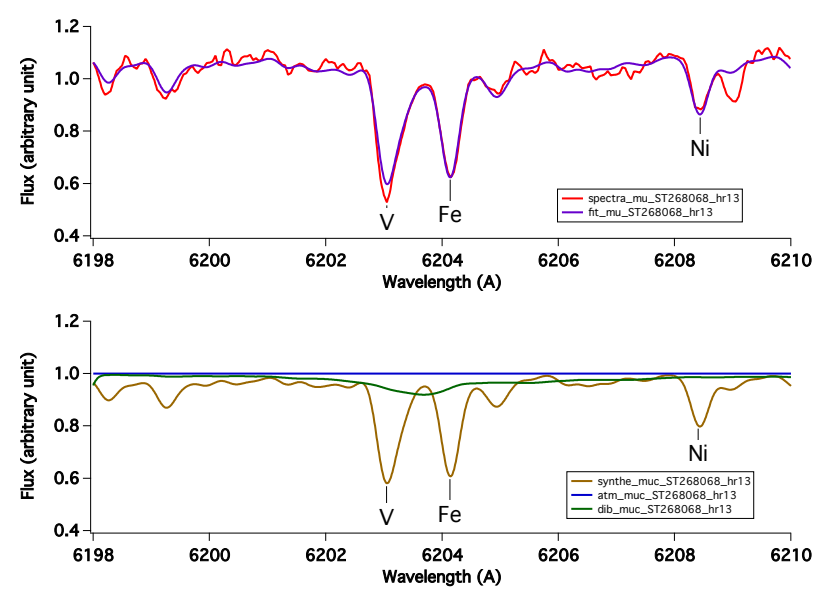

Fig. 9. Same as Fig. 8 for Ogle N: $268068\left(T_{\text {eff }}=4837 \mathrm{~K}\right)$. The DIB region corresponds to strong stellar lines.

are expected in future from elaborated studies of the stellar spectra in the DIB spectral regions, i.e. individual adjustments of the $\log (g f)$, studies of the missing lines, non-LTE and granulation effects. Such studies are beyond the scope of this work, which is devoted to testing the new method adapted to cool stars.

\section{DIB equivalent widths, DIB-DIB, and DIB-extinction correlations}

Table 3 lists the resulting EWs for the three DIBs, both before and after the empirical correction, as well as the associated uncertainty. Uncertainties, whose derivations are also described in detail in the Appendix, are a combination of random errors associated to the noise level and of errors linked to the use of the three models that remain after applying the above-mentioned empirical correction. These quasi-random uncertainties were derived from the whole set of residual vs metallicity curves that were computed for each wavelength and used for correcting of systematics. They were simply taken as the variance of the residuals around the mean relationship (see Figs. A.1 and A.2). This variances includes both the noise and the departure from the empirical ideal relationship between the metallicity and the stellar lines. It is clear that those derived uncertainties provide an order

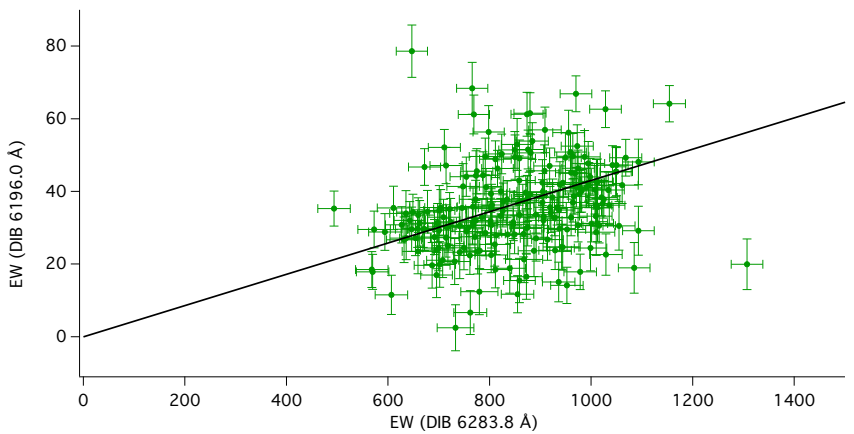

Fig. 10. Equivalent width (EW) of DIB 6196.0 $\AA$ as a function of the EW of DIB $6283.8 \AA$. The black line is the best linear fit for pure proportionality, using error bars of both DIBs.

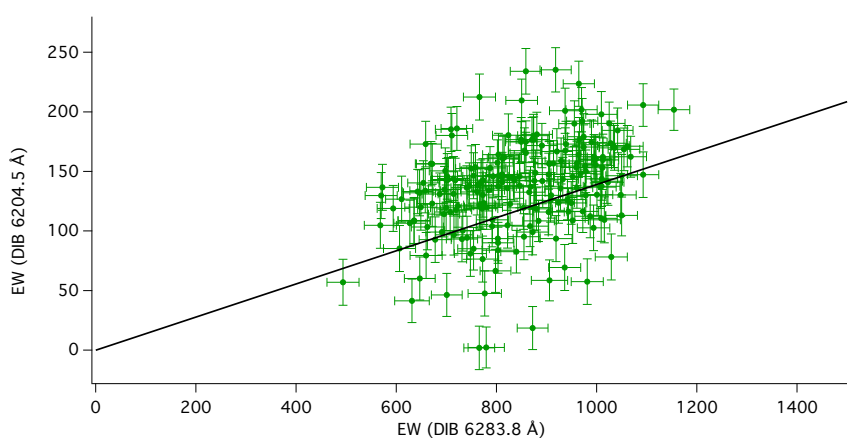

Fig. 11. Same as Fig. 10 for DIB $6204 \AA$.

of magnitude of the errors, which has a sense as a mean for all targets, but that individual errors for each target may be larger or smaller. Future work will address this point, once additional studies of the stellar models have been performed.

An immediate test of the reliability of the DIB strengths is the existence of DIB-DIB correlations, because EWs have been measured independently for the three bands. This test is not so easy here owing to the limited range of extinctions (Sumi 2004). Still, the Spearman's rank correlation statistics allows the absence of two-by-two correlations to be rejected by better than $99.9 \%$. Figures 10 and 11 show the DIBs 6196.0 and 6204.5 EWs as a function of the broad $6283.8 \AA \mathrm{EW}$ for the whole sample. The black lines correspond to a pure proportionality. Despite the large uncertainties, especially for the two small DIBs, the correlation between the three bands is clearly visible. Those correlations show that the signal we extracted for the DIBs contains some information on the IS absorption, even for the two small DIBs. A second test of the DIB measurement is obtained from a comparison with the extinction map. Figure 12 displays the star-by-star variations in each DIB EW, as well as the extinction derived from the OGLE photometry obtained by interpolation through the $A_{v}$ map of Sumi (2004). The four patterns, which represent variations across the field of the four independent quantities, reveal large similarities for the strong DIB and some similarities for the $6204 \AA$ DIB. Large uncertainties make the comparison less obvious for the $6196 \AA$ band. This is reflected in the Spearman's rank correlation statistics that allow rejecting the absence of correlation at better than $99.9 \%$ for the 6284 and 6204 DIBs, and 90\% for the $6196 \AA$ DIB.

More precise statistics could have been obtained after rejections of the noisy spectra or particularly poor adjustments. However, our goal here is to provide some idea of the DIB 

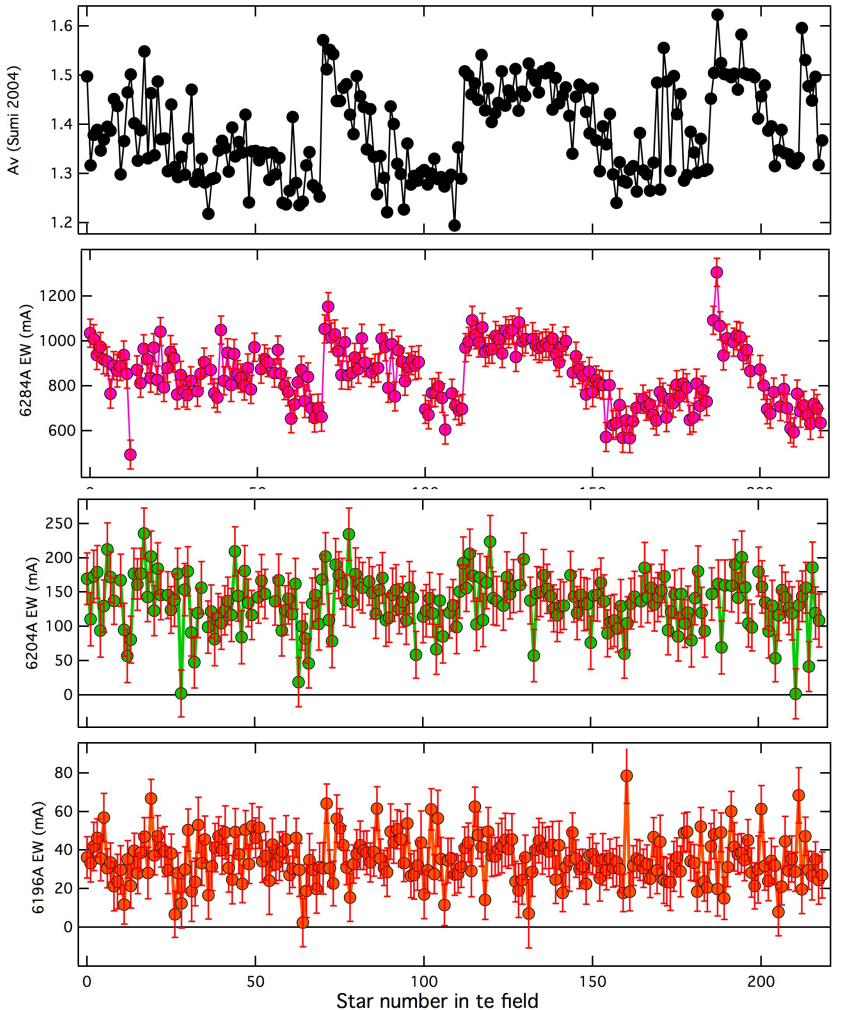

Fig. 12. From bottom to top, EWs of the three DIBs (6196, 6204, and $6284 \AA$ ) as a function of the star number and the extinction $A_{v}$ interpolated from the map of Sumi (2004) at each star location.

extraction results as a function of the signal quality, the DIB strength, and the reddening for an entire field, with an entirely automated method.

\section{Color excess estimates based on nearby star empirical relationships}

Diffuse interstellar bands are moderately or only weakly correlated with the extinction, as shown by a number of studies and the existence of DIB families whose members are internally closely correlated, while distinct families seem to obey different laws (e.g., Krelowski \& Walker 1987; Cami et al. 1997). There is no parental link between the three DIBs we study here, and thus we do not expect to find strong correlations between their EWs or very similar dependencies on the extinction. Previous surveys of stars in the solar neighborhood have provided some statistical relationships between the reddening $E_{B-V}$ derived from spectrophotometry and the EW of each DIB. Bestfit parameters of linear correlations have been established recently by Friedman et al. (2011) for all three DIBs, based on about 130 O-B nearby northern hemisphere stars within $1 \mathrm{kpc}$. In the case of the $6284 \AA$ DIBs, Raimond et al. (2012) derived slightly different coefficients based on about the same number of southern hemisphere targets, observed with the ESO/La Silla FEROS spectrograph. Using the same FEROS data, Puspitarini et al. (2012, priv. comm.) derived linear fit coefficients for the two other DIBs, again found to be slightly different when compared to the northern survey study. The coefficients for the linear relations between EW and $E_{B-V}$ are shown in Table 2 for both surveys and for the three DIBs. There are some differences between the relationships as they emerge from the two surveys, which have been discussed by Raimond et al. (2012). Owing to
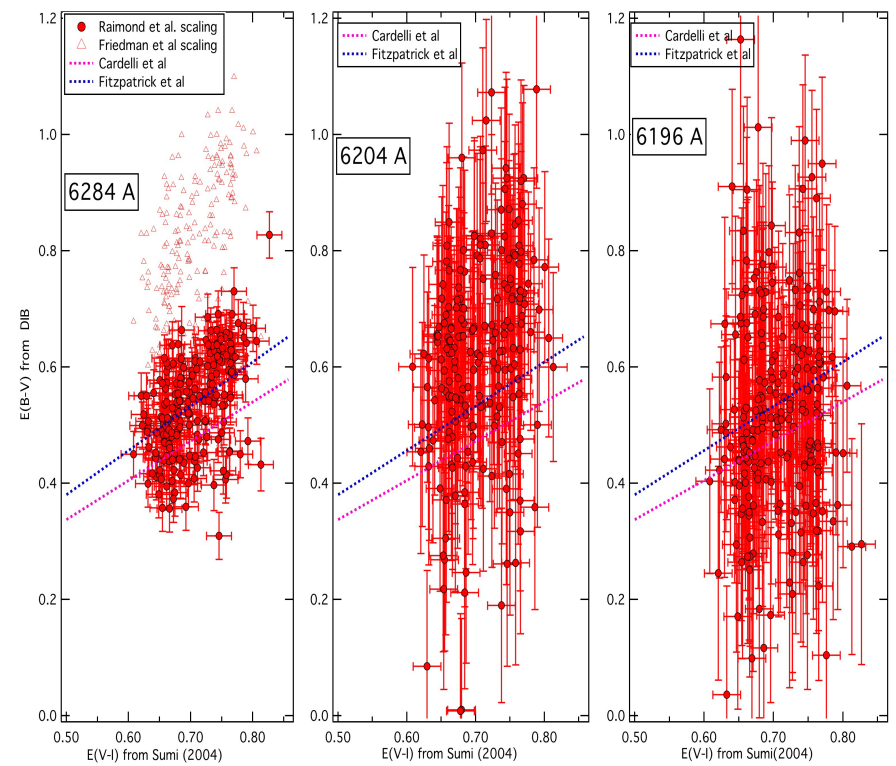

Fig. 13. Color excess $E_{B-V}$ derived from the three DIBs as a function of the $E_{V-I}$ value interpolated within the Sumi (2004) map at the locations of the target stars. $E_{B-V} \mathrm{~S}$ here are obtained using the Raimond et al. (2012) and Puspitarini et al. (2012, priv. comm.) average relationships based on FEROS data. The blue and pink dotted lines correspond to color excess relationships based on the Fitzpatrick (1999) and Cardelli et al. (1989) extinction curves and $R_{v}=3.1$ (see text). In the case of the DIBs $6283.8 \AA$ we also show the color excess values deduced from the Friedman et al. (2011) average relationships (open triangles).

Table 2. Value of $E_{B-V, \lambda}=a+b \times E W_{\lambda}$.

\begin{tabular}{lccc}
\hline \hline $\mathrm{DIB}_{\lambda}$ & Reference & $a$ & $b$ \\
\hline 6196.0 & 1 & $(-5.07 \pm 0.56) \times 10^{-2}$ & $(2.11 \pm 0.02) \times 10^{-2}$ \\
& 2 & 0 & $(1.48 \pm 0.09) \times 10^{-2}$ \\
6204.5 & 1 & $(-7.22 \pm 0.67) \times 10^{-2}$ & $(5.99 \pm 0.08) \times 10^{-3}$ \\
& 2 & 0 & $(4.58 \pm 0.37) \times 10^{-3}$ \\
6283.8 & 1 & $(-7.71 \pm 0.78) \times 10^{-2}$ & $(9.57 \pm 0.17) \times 10^{-4}$ \\
& 3 & $(-0.50 \pm 0.40) \times 10^{-2}$ & $(6.37 \pm 0.23) \times 10^{-4}$ \\
\hline
\end{tabular}

References. 1: Friedman et al. (2011); 2: Puspitarini et al. (2012, priv. comm.); 3: Raimond et al. (2012).

the use of cooler and fainter target stars, the ESO/FEROS DIB strengths are not as influenced by strong radiation fields, and there is significantly less dispersion around the mean DIB- $E_{B-V}$ relationship. As a matter of fact, DIB strengths may be significantly reduced in case the main dust cloud responsible for the absorption is very close to the UV-bright target star (Friedman et al. 2011; Vos et al. 2011), an effect attributed to the ionization state change of the carriers in the stellar environment. For this reason, the number of outliers with a relatively weak DIB and a large reddening is smaller in the FEROS survey, and the dispersion decreases. In parallel, the mean slope $E_{B-V} / \mathrm{EW}(\mathrm{DIB})$ derived from the sample is also smaller. This was found systematically for the three DIBs. Here we make use of the FEROSbased mean relationships, because the line-of-sight here is not related to hot bright stars and the DIB should correspond better to average conditions. For comparisons we also show both results for the strong $6284 \AA$ band.

The measured DIB EWs derived from the global fitting were converted into $E_{B-V}$ color excess values by application of the three average relationships listed in Table 2. 
Figure 13 shows the color excess $E_{B-V}$ estimate based on the DIB $6283.8 \AA$ and the FEROS relationship, as a function of the color excess $E_{V-I}$ interpolated from the Sumi (2004) maps ( $x$-axis). The figure is somewhat redundant with the previous one, however it provides a better idea of the EW dispersion and the global relationship. The data point distribution shows a correlation between the DIB-based and photometric determinations, but there is a large dispersion, and also the observed interval for the DIB strength is significantly larger than the range of variation in the photometric determination. (The best fit linear relationship does not go through zero.) The relationship between $E_{B-V}$ and $E_{V-I}$ depends on the extinction curve, and to a lesser extent on the stellar spectrum. We have drawn here the relationships based on both classical Cardelli et al. (1989) and Fitzpatrick (1999) extinction curves, for the total-to-selective extinction ratio $R_{v}=3.1$. The value of $E_{V-I}$ is computed for a typical redclump giant star, and $E_{B-V}$ is computed for the A0 star Vega. As far as average absolute values are concerned, the DIB-based $E_{B-V} \mathrm{~S}$ are similar to what one would expect from those classical laws, and in better agreement with the Fitzpatrick relationship. We also show the $E_{B-V}$ values we would obtain with the empirical Friedman et al. (2011) relationship. Those $E_{B-V}$ empirical estimates are systematically higher and more difficult to reconcile with the photometric data. We believe that the relationship based on the cooler FEROS targets may be more appropriate here because it probes environmental conditions that are closer to the average conditions encountered here than to the high ionization conditions encountered towards O- and early B-type nearby target stars.

Figure 13 also shows how the 6196 and $6204 \AA$ DIB-based color excess $E_{B-V}$ compares with the photometric determination of $E_{V-I}$. For those two DIBs, the correlation is visible, and the agreement between the measured and the expected ratio between the two color excess values is again better with the Fitzpatrick (1999) reddening law. There appears to be less deviation from average simple proportionality for those DIBs, compared to $6284 \AA$, especially for the DIB $6196 \AA$ for which the best-fit relationship is compatible with proportionality within the uncertainty range.

An important source of dispersion is the fact that the Sumi color excess is an average over the series of stars contained within the $0.6 \times 0.6$ arcmin pixel field and located at various distances in the bulge, while the DIB is derived for individual stars. Here the main effect would be angular variability, because, as said above, the quasi-totality of the extinction is generated closer than $2000 \mathrm{pc}$, thus differences in the distances to the bulge targets have in principle no impact. At $1 \mathrm{kpc}, 0.6$ acrimony corresponds to a transverse distance of $0.17 \mathrm{pc}$, thus only very dense, small cloud cores could produce variations as large as those observed. A likely explanation for both the dispersion and also departures from proportionality between the DIB-based color excess and the photometric $E_{V-I}$ is the existence of intrinsic DIB variations in response to the radiation field and in general to environmental conditions, such as those found at smaller distances. This is favored by the spatial pattern as we discuss below. On the other hand, spatial variations of the DIB- $E_{V-I}$ ratio may also be related to spatial variability of the extinction curve. Udalski (2003) and Sumi (2004) both consider $R_{v}$ may vary significantly towards the bulge. A relationship between the extinction curve, the total to selective extinction ratio and the DIB behavior has been first discussed by Krełowski et al. (1999). Environmental effects and dust processing, on one hand, and extinction curve, on the other, are themselves related through the grain distribution
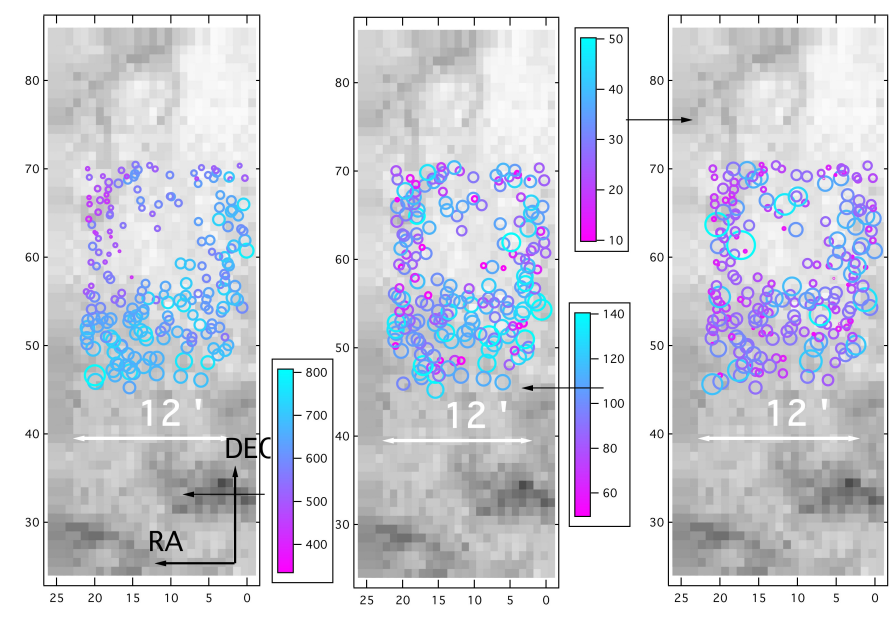

Fig. 14. Spatial variability of the DIB and the DIB-extinction ratio (from left to right, the $6284,6204,6196 \AA$ DIBs). Target stars used in this study are superimposed on the $A_{v}$ extinction map from Sumi (2004) (gray scale). The size of the circle is proportional to the DIB EW, and its color corresponds to the ratio between the DIB EW and the extinction $A_{v}$ obtained by interpolation through the Sumi map (color scale).

and the cloud history, which also influences DIB carriers and strengths.

The spatial variability of the ratio between DIB-based and photometric determinations of the color excess is shown superimposed on the Sumi map in Fig. 14. In this figure the color indicates the ratio and the size of the markers is proportional to the DIB strength. The distribution of colors shows that there are some systematic changes across the field for the 6284 DIB; in particular, the DIB is relatively small compared to the photometric value at larger declinations and right ascensions. Future investigations will hopefully allow those differences to be attributed in variations of the environmental conditions in the encountered clouds, to extinction law spatial variations, or both, as discussed above. The $6204 \AA$ DIB shows the same behavior as the 6284 band, although less clearly, due to large uncertainties and also not as pronounced. At variance with the other two bands, the $6196 \AA$ DIB does not reveal any spatial trend, although there are strong uncertainties on the DIB EW. If confirmed, for the three DIBs the spatial homogeneity of the measured ratio follows the degree of correlation of the DIBs with the color excess, as measured in the solar neighborhood and for widely distributed targets by Friedman et al. (2011). The correlation degree and spatial homogeneity decrease from the $6284 \AA$ DIB to the small $6196 \AA$ DIB.

Finally, we show in Fig. 15 a weighted mean value of $E_{B-V}$ derived from the three DIBs, again compared with the $E_{V-I}$ value deduced from the OGLE analysis. This figure allows determining to what extent the combination of those three DIBs can be used here as a first estimator for the reddening, in the absence of photometric measurements. The average linear relationship is $E_{B-V}=(0.72 \pm 0.14) E_{V-I}+(0.06 \pm 0.10)$ and the standard deviation is 0.08 , i.e. on the order of $15 \%$ of the average value. This should be compared with other conditions and distances.

\section{Summary and discussion}

We have used $R=22500, S / N=30-77$ observations of 219 red clump giants from the galactic bulge in Baade's Window $\left(A_{v} \simeq 1.4\right)$ as a test case for our newly developed composite 


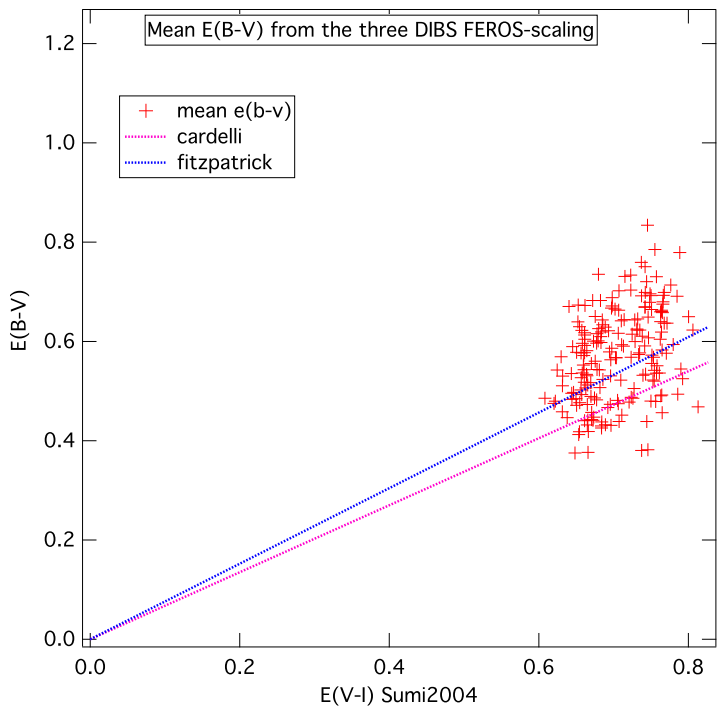

Fig. 15. Synthesis of all $E_{B-V}$ s estimates from the 3 DIBs, in the form of a weighted mean, here compared with the $E_{V-I}$ determination from the OGLE photometry. The blue and pink dotted lines correspond to color excess relationships based on the Fitzpatrick (1999) and Cardelli et al. (1989) extinction curves.

model and automated fitting method of IS absorption extraction from cool star spectra. The combination of synthetic stellar models, synthetic atmospheric lines, and DIB profiles allowed us to extract EW values for the three DIBs 6284, 6196, and $6204 \AA$. The existence of DIB-DIB correlations demonstrates that, even without any adaptation of the stellar model, in the case of a moderate color excess $E_{B-V} \simeq 0.4$ and of signal-to-noise ratios above 30, a strong DIB like the $6284 \AA$ band, but also narrow and weaker DIBs like the 6196 or the $6204 \AA$ bands, can be measured for cool stars, provided one takes the velocity shift into account between the star and the absorbing ISM. More precisely, all spectra could be efficiently used in the case of the strong and broad $6284 \AA$ DIB (width $\simeq 3.5 \AA$, depth $\simeq 20 \%$ ), while for the two narrow and weaker DIBs $(\simeq 0.5$ and $1 \AA$ width, $10 \%$ depth $)$ the potential extraction of the DIB EW depends on the star's radial velocity, whose value results in an overlap of stellar lines and DIBs or does not. This is due to the presence of unidentified lines, as well as to over- or underpredicted line strengths. Model improvements are beyond the scope of this work, but should be performed in future, once other analyses confirm the present trends and enough constraints have been obtained. Here we simply performed an empirical correction, independently for each DIB, which is described in the Appendix. Its validity was demonstrated by a strong improvement in the DIB-DIB correlations, as well as improvements in the DIB-extinction correlations. More work is needed on a more fundamental approach to those corrections. Overall, this modeling demonstrates that DIBs can be measured in an automated way for a large number of cool targets during spectroscopic surveys, and be used as any other IS line to locate the IS matter.

For the three DIBs, the spatial pattern generally reflects extinction variations deduced from stellar photometry, which also validates the fitting method. The degree of correlation is, as expected, generally better for the broader and stronger DIB because uncertainties on the EWs are much smaller. DIB strengths are converted into color excesses, using best-fit linear relationships established for early-type star surveys. Mean values of
$E_{B-V}$ over all targets are $0.53,0.62$, and 0.52 for the 6284, 6204, and $6196 \AA$ DIBs, respectively. Discrepancies among the DIBs amount to about $20 \%$, and there is a $15 \%$ dispersion of the weighted mean value around its average linear relationship with the photometric reddening (Fig. 15). There were several results from the analysis that call for further studies. The 6284, and to a smaller extent the $6204 \AA$ DIBs amplitude intervals over the field, are found to be larger than the color excess relative variations deduced from OGLE photometric data and their analysis (Sumi 2004, see Fig. 12). This is reflected in the spatial variability of the DIB-based to photometry-based color excess ratio. Those variations are not randomly located, as is especially visible for the $6284 \AA$ DIB: the DIB is systematically weaker around $\alpha, \delta=270.95,-30^{\circ}$. One likely explanation for such departures from proportionality is the DIB response to ionization conditions in the clouds and other environmental effects such as shocks. This may be linked to the spatial variability of the extinction law. The grain size distribution influences the shape of the extinction law and the $R_{v}$, but it is also linked to the cloud's physical properties and its history, which in turn influence the quantity of macro-molecules and the DIBs. Such a link between the $R_{v}$ and the DIBs has been discussed by Krełowski et al. (1999). The excess of amplitude variation and the spatial variability do not seem to exist for the small $6196 \AA$ DIB, although large uncertainties make the comparison with the photometric extinction more difficult. This may relate to this DIB having been found to be better correlated with the extinction than the two others, something again potentially related to its different response to the dust distribution, or the environmental conditions. Moreover, the absolute value of the extinction based on the $6196 \AA$ DIB (again deduced from the average solar neighborhood relationship) seems to agree better with the photometric determination than do the two other DIBs.

More analyses should be performed over various fields and for targets at various distances to confirm unidentified or poorly predicted stellar lines. More data should help in refining the stellar models in the DIB spectral regions and subsequently improve the DIB extraction. On the other hand, extinction estimates would certainly be strongly improved by the use of multiple DIBs and of their ratios, accompanied by better understanding of their specific behavior. Such studies may also provide additional information on the links between the extinction law and the DIBs.

Acknowledgements. We thank the referee for the useful comments on the manuscript. H.-C. C. wants to acknowledge the Taiwanese government for her scholarship NSC100-2917-I-564-057.

\section{Appendix A: Error estimates}

Estimating uncertainties on the DIB EWs is not straightforward here, as there are different sources of errors. The error linked to measurement uncertainties can in principle be classically estimated. For the 219 spectra of this study, the signal-to-noise ratio varies between from 30 and 77 per pixel. It can be immediately derived that for a broad DIB covering about $6 \AA$ (i.e. defined over more than 100 pixels and of about $10-15 \%$ depth, which corresponds to the $6284 \AA$ one), the relative error due to the noise should only be very small. For the shallower and narrower DIBs, this is not the case. The other major source of uncertainties comes from the use of synthetic spectra, which have not been adjusted individually for all targets. As shown by Figs. 6 to 9, those uncertainties may approach the DIB itself depending on the star radial velocity, for the two small DIBs. 


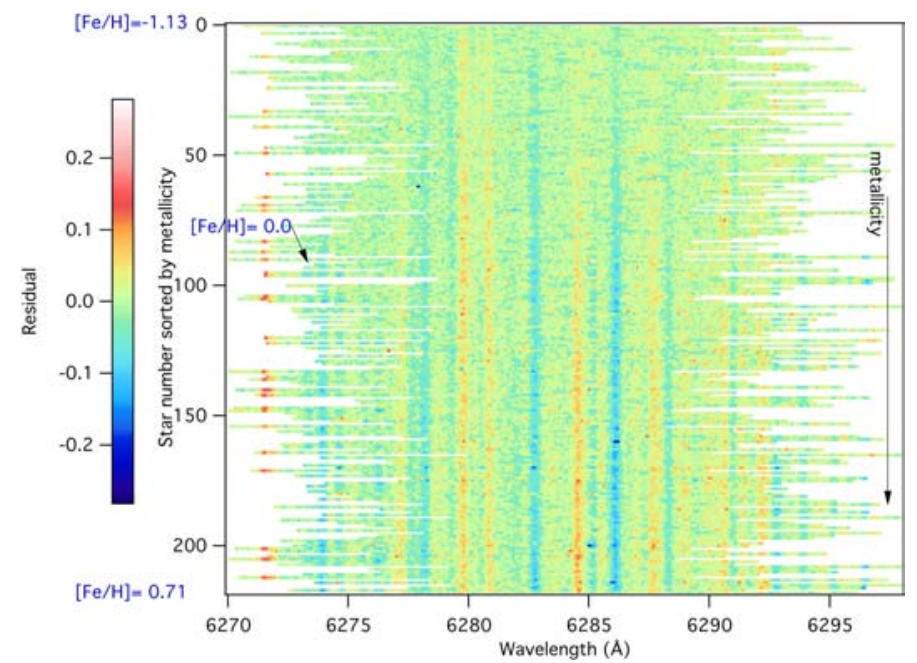

Fig. A.1. Residuals as a function of wavelength and metallicity for the DIB $6283.8 \AA$. They form the basis for the EW correction and the error estimate.

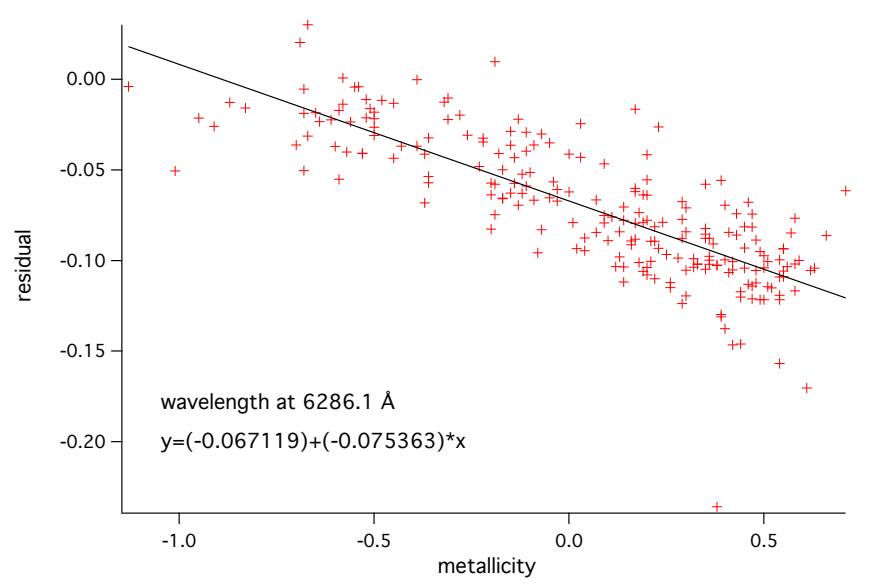

Fig. A.2. Example of residuals vs. -metallicity relationships: here the residuals depend on the metallicity and are negative, which corresponds to an under-predicted stellar absorption line.

We have devised a method for error estimates that has the advantage of providing a corrective term for the DIB EW in addition to the errors. For each target, the (data - model) residuals for the best adjustment are shifted into the stellar frame. Those residuals are then sorted by metallicity. The corresponding 2D residual plot is shown in Fig. A.1 for the $6284 \AA$ DIB. The $x$-axis is the wavelength in the star frame, and the $y$-axis is the metallicity. The color scale represents the value of the residuals. Obviously there are some features at specific wavelengths, some quite strong, and they are also obviously related to the metallicity. They correspond to stellar lines that are underor overpredicted by the synthetic spectra. A large number of lines are simply unidentified. The next step is to use this map in the orthogonal direction, i.e., to extract the residual as a function of the metallicity, for each wavelength in the star frame. Examples of the obtained extracted series are shown in Figs. A.2 and A.3. Each of those metallicity-residual series of points is then fitted to a linear relationship, as shown in the two plots. For the wavelength corresponding to Fig. A.2, the residual is negative, and its absolute value increase strongly with the metallicity. This means that at this location there is a stellar line that is under-predicted and that the higher the metallicity, the greater

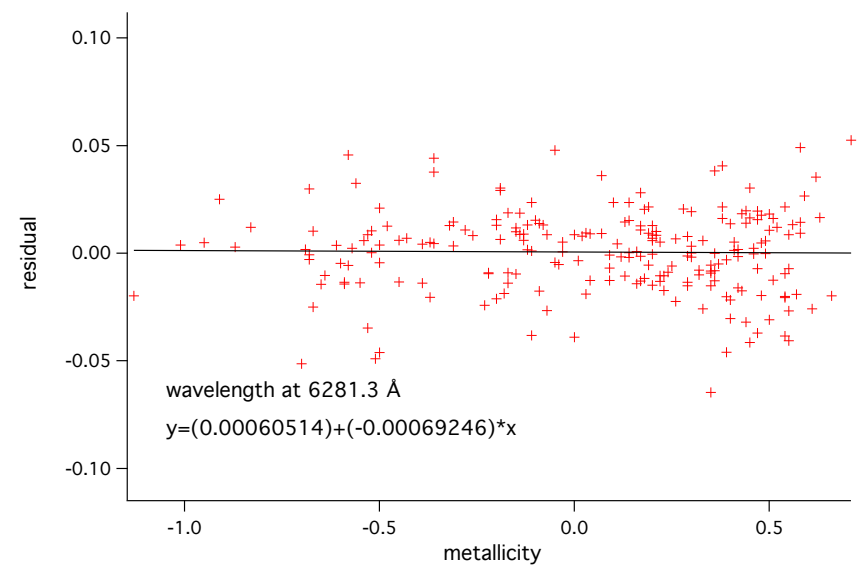

Fig. A.3. Example of residuals vs. -metallicity relationships: here the residuals do not depend on the metallicity and are negligible.

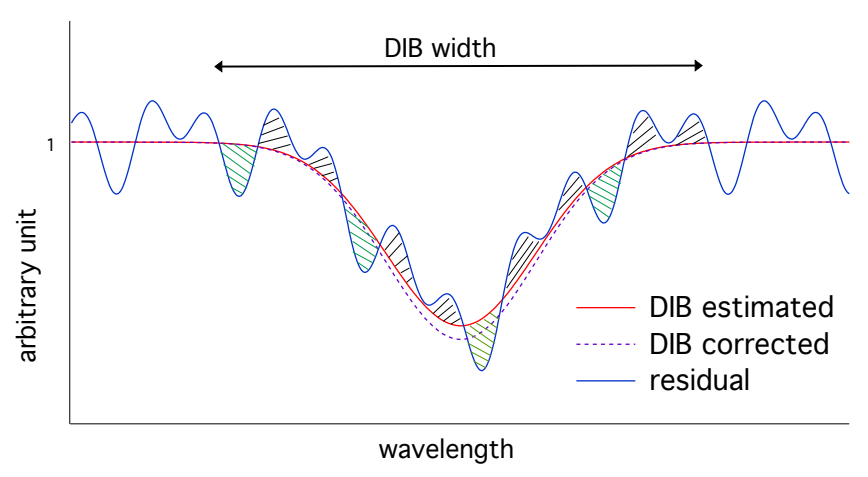

Fig. A.4. Schematic illustration of the correction applied to the EW measurements. The red curve represents the DIB profile as it comes out from the fitting phase. Hatched surfaces show the offsets applied at each wavelength that are computed for each star, as a function of its metallicity. The dashed line illustrates the corrected DIB.

the data-model discrepancy. At variance with this case, at the wavelength corresponding to Fig. A.3, the residual is on average zero and not related to the metallicity. This means that there is no stellar line at this location, or that the model does predict the line adequately. We then use both the fit coefficients (at each wavelength) to correct the previously computed DIB profile and the standard deviation around the mean relationship (again at each wavelength) to estimate the random (or quasi-random) remaining errors. More precisely, for each star we consider the wavelength interval that contains the entire DIB, and compute for each wavelength the most-probable residual as a function of the star metallicity, based on the linear relationships coefficients. We apply this correction over the whole DIB interval (as shown in Fig. A.4) and recalculate the DIB EW. Table 3 contains both the initial and corrected values for the EW for the three DIBs and all targets. We found that the recalculated EWs provide significantly improved DIB-DIB correlations, which demonstrates that this method provides a partial, but valid correction.

The distribution of data points around the mean residualmetallicity relationship provides an estimate of the combination of unpredictable uncertainties linked to the stellar lines and the actual noise). We then used the standard deviation measured for each relationship to compute the standard deviation at each wavelength, then propagated the errors on the whole DIB interval. In the case of the two narrow DIBs, this estimated uncertainty may be smaller in some cases than the actual errors; 
H.-C. Chen et al.: Extracting interstellar diffuse absorption bands from cool star spectra

however, considering those deviations as systematic and not random leads to errors that are unrealistically large.

\section{References}

Cami, J., Sonnentrucker, P., Ehrenfreund, P., \& Foing, B. H. 1997, A\&A, 326, 822

Cardelli, J. A., Clayton, G. C., \& Mathis, J. S. 1989, ApJ, 345, 245

Clough, S. A., Shephard, M. W., Mlawer, E. J., et al. 2005, J. Quant. Spec. Radiat. Transf., 91, 233

Fitzpatrick, E. L. 1999, PASP, 111, 63

Friedman, S. D., York, D. G., McCall, B. J., et al. 2011, ApJ, 727, 33

Fulara, J., \& Krelowski, J. 2000, New Astron. Rev., 44, 581

Galazutdinov, G. A., Lo Curto, G., \& Krełowski, J. 2008, MNRAS, 386, 2003

Herbig, G. H. 1995, ARA\&A, 33, 19

Hill, V., Lecureur, A., Gómez, A., et al. 2011, A\&A, 534, A80

Hobbs, L. M., York, D. G., Snow, T. P., et al. 2008, ApJ, 680, 1256

Hobbs, L. M., York, D. G., Thorburn, J. A., et al. 2009, ApJ, 705, 32

Jenniskens, P., \& Desert, F.-X. 1994, A\&AS, 106, 39

Krelowski, J., \& Walker, G. A. H. 1987, ApJ, 312, 860

Krełowski, J., Ehrenfreund, P., Foing, B. H., et al. 1999, A\&A, 347, 235
Kurucz, R. L. 2005, Mem. Soc. Astron. It. Suppl., 8, 14

Lallement, R., Bertin, P., Chassefiere, E., \& Scott, N. 1993, A\&A, 271, 734

Marshall, D. J., Robin, A. C., Reylé, C., Schultheis, M., \& Picaud, S. 2006, A\&A, 453, 635

Moore, C. E., Minnaert, M. G. J., \& Houtgast, J. 1966, National Bureau of Standards Monograph, Washington: US Government Printing Office (USGPO)

Raimond, S., Lallement, R., Vergely, J. L., Babusiaux, C., \& Eyer, L. 2012, A\&A, 544, A136

Rothman, L. S., Gordon, I. E., Barbe, A., et al. 2009, J. Quant. Spec. Radiat. Transf., 110, 533

Salama, F., Bakes, E. L. O., Allamandola, L. J., \& Tielens, A. G. G. M. 1996, ApJ, 458, 621

Sbordone, L. 2005, Mem. Soc. Astron. It. Suppl., 8, 61

Sbordone, L., Bonifacio, P., Castelli, F., \& Kurucz, R. L. 2004, Mem. Soc. Astron. It. Suppl., 5, 93

Snow, T. P., \& Destree, J. D. 2011, EAS Publ. Ser., 46, 341

Sumi, T. 2004, MNRAS, 349, 193

Udalski, A. 2003, ApJ, 590, 284

Vos, D. A. I., Cox, N. L. J., Kaper, L., Spaans, M., \& Ehrenfreund, P. 2011, A\&A, 533, A129 
Table 3. Stellar data and measurement of EW.

\begin{tabular}{|c|c|c|c|c|c|c|c|c|c|c|c|c|}
\hline Star & OgleN & $\begin{array}{c}T_{\mathrm{eff}} \\
\mathrm{K}\end{array}$ & $\begin{array}{c}\log g \\
\text { Dec }\end{array}$ & $\begin{array}{c}\xi \\
\mathrm{km} \mathrm{s}^{-1}\end{array}$ & $\begin{array}{c}{[\mathrm{Fe} / \mathrm{H}]} \\
\mathrm{Dec}\end{array}$ & $E_{B-V^{*}}$ & $\begin{array}{c}\mathrm{EW}(6196) \\
\mathrm{mA}\end{array}$ & $\begin{array}{c}\text { Corr'd } \\
\text { mA }\end{array}$ & $\begin{array}{c}\mathrm{EW}(6204) \\
\mathrm{mA}\end{array}$ & $\begin{array}{c}\text { Corr'd } \\
\mathrm{m} \AA\end{array}$ & $\begin{array}{c}\mathrm{EW}(6284) \\
\mathrm{m} \AA\end{array}$ & $\begin{array}{c}\text { Corr'd } \\
\mathrm{m} \AA\end{array}$ \\
\hline 1 & 67536 & 5121 & 2.43 & 1.1 & -0.45 & 0.483 & 40 & $36 \pm 11$ & 164 & $170 \pm 38$ & 1046 & $1036 \pm 62$ \\
\hline 2 & 67610 & 4865 & 2.34 & 1.5 & -0.31 & 0.425 & 36 & $33 \pm 10$ & 110 & $111 \pm 38$ & 1053 & $1009 \pm 64$ \\
\hline 3 & 67641 & 4575 & 2.17 & 1.4 & -0.36 & 0.445 & 50 & $42 \pm 13$ & 164 & $173 \pm 38$ & 962 & $939 \pm 64$ \\
\hline 4 & 67663 & 4878 & 2.33 & 1.4 & -0.05 & 0.448 & 50 & $46 \pm 10$ & 175 & $179 \pm 39$ & 1023 & $974 \pm 64$ \\
\hline 5 & 67675 & 4507 & 2.16 & 1.5 & 0.42 & 0.434 & 49 & $36 \pm 12$ & 87 & $94 \pm 38$ & 991 & $919 \pm 64$ \\
\hline 6 & 67687 & 4664 & 2.27 & 1.5 & 0.54 & 0.442 & 64 & $57 \pm 13$ & 130 & $130 \pm 38$ & 942 & $909 \pm 64$ \\
\hline 7 & 67727 & 4994 & 2.45 & 1.5 & 0.55 & 0.45 & 37 & $31 \pm 10$ & 206 & $213 \pm 38$ & 838 & $766 \pm 64$ \\
\hline 8 & 67744 & 5170 & 2.42 & 1.4 & -0.43 & 0.447 & 36 & $34 \pm 10$ & 175 & $172 \pm 37$ & 895 & $891 \pm 62$ \\
\hline 9 & 67762 & 4943 & 2.34 & 1.3 & -0.26 & 0.468 & 21 & $21 \pm 13$ & 131 & $137 \pm 34$ & 884 & $868 \pm 73$ \\
\hline 10 & 78185 & 4763 & 2.3 & 1.2 & 0.21 & 0.464 & 13 & $24 \pm 14$ & 105 & & 840 & $887 \pm 62$ \\
\hline 11 & 78195 & 4807 & 2.34 & 1.1 & -0.05 & 0.419 & 34 & $30 \pm 13$ & 161 & $167 \pm 38$ & 951 & $938 \pm 62$ \\
\hline 12 & 78202 & 4460 & 2.17 & 1.5 & 0.54 & 0.441 & 19 & $12 \pm 10$ & 88 & $95 \pm 38$ & 924 & $855 \pm 64$ \\
\hline 13 & 78205 & 5271 & 2.37 & 1 & 0.2 & 0.473 & 39 & $35 \pm 10$ & 53 & $57 \pm 38$ & 555 & $493 \pm 64$ \\
\hline 14 & 78215 & 4738 & 2.22 & 1.6 & 0.55 & 0.484 & 38 & $22 \pm 12$ & 53 & $81 \pm 34$ & 747 & \\
\hline 15 & 78255 & 4810 & 2.31 & 1.4 & 0.49 & 0.452 & 38 & $40 \pm 9$ & 184 & $177 \pm 37$ & 852 & $871 \pm 63$ \\
\hline 16 & 78271 & 4685 & 2.21 & 1.5 & 0.17 & 0.428 & 24 & $28 \pm 11$ & 155 & $161 \pm 38$ & 816 & $813 \pm 62$ \\
\hline 17 & 78281 & 4899 & 2.31 & 1.1 & 0.2 & 0.448 & 32 & $39 \pm 10$ & 179 & $177 \pm 37$ & 945 & $966 \pm 63$ \\
\hline 18 & 78283 & 4512 & 2.21 & 1.5 & 0.35 & 0.5 & 48 & $47 \pm 10$ & 242 & $235 \pm 37$ & 895 & $918 \pm 63$ \\
\hline 19 & 78288 & 5136 & 2.3 & 1.5 & -0.48 & 0.429 & 27 & $28 \pm 13$ & 126 & $143 \pm 36$ & 800 & $835 \pm 63$ \\
\hline 20 & 78322 & 4978 & 2.37 & 0.8 & -0.67 & 0.472 & 70 & $67 \pm 10$ & 205 & $202 \pm 37$ & 978 & $970 \pm 63$ \\
\hline 21 & 78323 & 4867 & 2.35 & 1.5 & 0.3 & 0.431 & 27 & $37 \pm 10$ & 131 & $123 \pm 36$ & 799 & $827 \pm 63$ \\
\hline 22 & 78330 & 4595 & 2.18 & 1.5 & 0.61 & 0.48 & 40 & $47 \pm 11$ & 180 & $185 \pm 37$ & 1035 & $1042 \pm 62$ \\
\hline 23 & 78354 & 4738 & 2.29 & 1 & 0.03 & 0.442 & 35 & $41 \pm 10$ & 152 & $146 \pm 36$ & 768 & $793 \pm 63$ \\
\hline 24 & 78357 & 4620 & 2.09 & 1.5 & 0.37 & 0.442 & 29 & $39 \pm 13$ & 136 & & 840 & $880 \pm 63$ \\
\hline 25 & 78379 & 4775 & 2.31 & 1.3 & 0.03 & 0.421 & 34 & $30 \pm 12$ & 131 & $146 \pm 34$ & 960 & $952 \pm 73$ \\
\hline 26 & 78393 & 4943 & 2.26 & 1.2 & -0.18 & 0.465 & 33 & $38 \pm 10$ & 133 & $124 \pm 34$ & 903 & $924 \pm 63$ \\
\hline 27 & 78401 & 4851 & 2.24 & 1.5 & 0.14 & 0.424 & 11 & $7 \pm 12$ & 140 & $138 \pm 38$ & 818 & $762 \pm 64$ \\
\hline 28 & 78421 & 4632 & 2.22 & 1.5 & 0.46 & 0.417 & 25 & $28 \pm 11$ & 177 & $177 \pm 37$ & 838 & $849 \pm 62$ \\
\hline 29 & 78436 & 4541 & 2.19 & 1.3 & 0.32 & 0.43 & 23 & $12 \pm 13$ & -21 & $2 \pm 34$ & 802 & $780 \pm 73$ \\
\hline 30 & 78449 & 4947 & 2.22 & 1.7 & 0.54 & 0.418 & 25 & $30 \pm 12$ & 155 & $154 \pm 38$ & 759 & $758 \pm 62$ \\
\hline 31 & 78461 & 4539 & 2.16 & 1.3 & 0.32 & 0.442 & 37 & $50 \pm 11$ & 218 & $180 \pm 36$ & 809 & $824 \pm 63$ \\
\hline 32 & 89573 & 4819 & 2.25 & 1.4 & 0.35 & 0.474 & 44 & $19 \pm 16$ & 74 & $91 \pm 34$ & 885 & \\
\hline 33 & 89589 & 4690 & 2.13 & 1.5 & -0.13 & 0.414 & 25 & $24 \pm 12$ & 48 & $48 \pm 38$ & 817 & $777 \pm 67$ \\
\hline 34 & 89590 & 5151 & 2.42 & 1.5 & -0.23 & 0.419 & 37 & $53 \pm 14$ & 115 & $120 \pm 36$ & 798 & $854 \pm 62$ \\
\hline 35 & 89609 & 4898 & 2.29 & 1.2 & -0.15 & 0.429 & 35 & $33 \pm 12$ & 149 & $157 \pm 38$ & 915 & $906 \pm 62$ \\
\hline 36 & 89614 & 4734 & 2.26 & 1.5 & 0.59 & 0.414 & 39 & $46 \pm 12$ & 76 & & 784 & \\
\hline 37 & 89640 & 4641 & 2.21 & 1.5 & 0.19 & 0.393 & 18 & $17 \pm 12$ & 97 & $99 \pm 38$ & 878 & $872 \pm 62$ \\
\hline 38 & 89645 & 4674 & 2.22 & 1.6 & 0.28 & 0.416 & 41 & $32 \pm 13$ & 118 & $122 \pm 38$ & 797 & $767 \pm 64$ \\
\hline 39 & 89667 & 4516 & 2.11 & 1.5 & 0.66 & 0.417 & 53 & $41 \pm 13$ & 78 & $81 \pm 38$ & 783 & $748 \pm 64$ \\
\hline 40 & 89669 & 4964 & 2.44 & 1.4 & 0.39 & 0.434 & 35 & $47 \pm 10$ & 141 & $113 \pm 34$ & 1029 & $1050 \pm 63$ \\
\hline 41 & 89687 & 4743 & 2.33 & 1.5 & 0.5 & 0.441 & 43 & $40 \pm 13$ & 106 & $105 \pm 38$ & 846 & $822 \pm 63$ \\
\hline 42 & 89702 & 4714 & 2.2 & 1.2 & 0.2 & 0.436 & 31 & $49 \pm 14$ & 148 & $124 \pm 36$ & 913 & $949 \pm 62$ \\
\hline 43 & 89731 & 4784 & 2.22 & 1.3 & -0.12 & 0.421 & 33 & $31 \pm 13$ & 129 & $137 \pm 35$ & 825 & $803 \pm 72$ \\
\hline 44 & 89735 & 4579 & 2.07 & 1 & 0.11 & 0.45 & 36 & $25 \pm 13$ & 111 & $116 \pm 38$ & 976 & $944 \pm 64$ \\
\hline 45 & 89736 & 4483 & 2.15 & 1.4 & 0.47 & 0.431 & 30 & $50 \pm 12$ & 255 & $210 \pm 36$ & 837 & $850 \pm 63$ \\
\hline 46 & 89774 & 4635 & 2.13 & 1.5 & 0.22 & 0.44 & 39 & $38 \pm 10$ & 150 & $145 \pm 37$ & 820 & $834 \pm 62$ \\
\hline 47 & 89786 & 4705 & 2.31 & 1.1 & -0.19 & 0.433 & 26 & $23 \pm 10$ & 82 & $84 \pm 39$ & 851 & $803 \pm 64$ \\
\hline 48 & 89802 & 4481 & 2.12 & 1.5 & 0.45 & 0.458 & 52 & $51 \pm 10$ & 188 & $181 \pm 37$ & 855 & $881 \pm 62$ \\
\hline 49 & 89832 & 4546 & 2.14 & 1.4 & 0.17 & 0.4 & 25 & $33 \pm 10$ & 150 & $134 \pm 35$ & 757 & $785 \pm 63$ \\
\hline 50 & 89843 & 5270 & 2.38 & 1.1 & 0.17 & 0.434 & 40 & $53 \pm 12$ & 144 & $116 \pm 36$ & 956 & $972 \pm 63$ \\
\hline 51 & 89848 & 4582 & 2.08 & 1.4 & 0.09 & 0.434 & 47 & $46 \pm 10$ & 72 & & 931 & \\
\hline 52 & 89871 & 4502 & 2.17 & 1.5 & 0.36 & 0.428 & 47 & $52 \pm 11$ & 138 & $142 \pm 37$ & 872 & $876 \pm 62$ \\
\hline 53 & 101453 & 4966 & 2.39 & 1.2 & -0.1 & 0.433 & 35 & $34 \pm 11$ & 160 & $167 \pm 38$ & 928 & $920 \pm 62$ \\
\hline 54 & 101462 & 4807 & 2.25 & 1.3 & 0.07 & 0.433 & 24 & $37 \pm 13$ & 167 & $147 \pm 36$ & 883 & $904 \pm 63$ \\
\hline 55 & 101470 & 4567 & 2.2 & 1.4 & 0.62 & 0.415 & 5 & $24 \pm 17$ & 22 & & 999 & \\
\hline 56 & 101476 & 4838 & 2.34 & 1.5 & -0.11 & 0.433 & 30 & $43 \pm 14$ & 110 & & 802 & $858 \pm 63$ \\
\hline 57 & 101486 & 4760 & 2.15 & 1.3 & -0.6 & 0.419 & 34 & $33 \pm 10$ & 135 & $136 \pm 35$ & 941 & $961 \pm 63$ \\
\hline 58 & 101505 & 4584 & 2.16 & 1.5 & 0.17 & 0.429 & 44 & $37 \pm 13$ & 164 & $168 \pm 38$ & 884 & $856 \pm 64$ \\
\hline 59 & 101540 & 4754 & 2.35 & 1.5 & 0.38 & 0.4 & 39 & $39 \pm 10$ & 98 & $94 \pm 37$ & 789 & $803 \pm 62$ \\
\hline 60 & 101606 & 4647 & 2.08 & 1.5 & 0.58 & 0.399 & 23 & $46 \pm 12$ & 175 & $123 \pm 36$ & 763 & $775 \pm 63$ \\
\hline 61 & 101638 & 4556 & 2.14 & 1.4 & -0.37 & 0.408 & 28 & $27 \pm 10$ & 145 & $140 \pm 37$ & 650 & $654 \pm 63$ \\
\hline 62 & 101648 & 5025 & 2.33 & 1.6 & -0.07 & 0.457 & 31 & $30 \pm 10$ & 124 & $118 \pm 37$ & 708 & $719 \pm 63$ \\
\hline 63 & 101650 & 4801 & 2.21 & 1.5 & 0.44 & 0.413 & 48 & $46 \pm 10$ & 169 & $162 \pm 37$ & 792 & $815 \pm 62$ \\
\hline
\end{tabular}

Notes. $* E_{B-V}$ is derived from the Sumi Sumi (2004) by interpolation. 
Table 3. continued.

\begin{tabular}{|c|c|c|c|c|c|c|c|c|c|c|c|c|}
\hline Star & OgleN & $\begin{array}{c}T_{\text {eff }} \\
\mathrm{K}\end{array}$ & $\begin{array}{l}\log g \\
\text { Dec }\end{array}$ & $\begin{array}{c}\xi \\
\mathrm{km} \mathrm{s}^{-1}\end{array}$ & $\begin{array}{c}{[\mathrm{Fe} / \mathrm{H}]} \\
\mathrm{Dec}\end{array}$ & $E_{B-V^{*}}$ & $\begin{array}{c}\mathrm{EW}(6196) \\
\mathrm{m} \AA\end{array}$ & $\begin{array}{c}\text { Corr'd } \\
\mathrm{m} \AA\end{array}$ & $\begin{array}{c}\mathrm{EW}(6204) \\
\mathrm{m} \AA\end{array}$ & $\begin{array}{c}\text { Corr'd } \\
\mathrm{m} \AA\end{array}$ & $\begin{array}{c}\mathrm{EW}(6284) \\
\mathrm{m} \AA\end{array}$ & $\begin{array}{c}\text { Corr'd } \\
\mathrm{m} \AA\end{array}$ \\
\hline 64 & 101655 & 5274 & 2.46 & 1.2 & 0.71 & 0.399 & 14 & $30 \pm 14$ & 76 & $19 \pm 36$ & 826 & $872 \pm 62$ \\
\hline 65 & 101662 & 4465 & 2.05 & 1.2 & 0.29 & 0.401 & 12 & $3 \pm 13$ & 80 & $100 \pm 35$ & 757 & $733 \pm 73$ \\
\hline 66 & 101674 & 4644 & 2.23 & 1.5 & 0.43 & 0.425 & -5 & $19 \pm 14$ & 122 & $83 \pm 36$ & 808 & $840 \pm 62$ \\
\hline 67 & 101681 & 4763 & 2.23 & 1.5 & 0.36 & 0.433 & 22 & $35 \pm 14$ & 82 & $46 \pm 36$ & 654 & $701 \pm 62$ \\
\hline 68 & 101691 & 4857 & 2.23 & 1.6 & 0.21 & 0.412 & 30 & $30 \pm 10$ & 137 & $134 \pm 37$ & 649 & $658 \pm 62$ \\
\hline 69 & 101692 & 4449 & 1.99 & 1.5 & 0.25 & 0.41 & 21 & $20 \pm 12$ & 145 & $146 \pm 38$ & 707 & $702 \pm 62$ \\
\hline 70 & 101703 & 4931 & 2.2 & 1.5 & 0.16 & 0.404 & 41 & $31 \pm 13$ & 99 & $103 \pm 38$ & 691 & $662 \pm 64$ \\
\hline 71 & 234759 & 4458 & 2.06 & 1.5 & 0.35 & 0.507 & 6 & $31 \pm 13$ & 204 & $169 \pm 36$ & 1028 & $1055 \pm 63$ \\
\hline 72 & 234802 & 4288 & 1.97 & 0.8 & 0.23 & 0.488 & 55 & $64 \pm 10$ & 229 & $202 \pm 35$ & 1135 & $1154 \pm 63$ \\
\hline 73 & 234810 & 4521 & 2.12 & 1.2 & -0.5 & 0.5 & 32 & $31 \pm 10$ & 111 & $109 \pm 39$ & 1054 & $1016 \pm 64$ \\
\hline 74 & 234811 & 4773 & 2.28 & 1.4 & -0.39 & 0.498 & 24 & $23 \pm 11$ & 81 & $78 \pm 39$ & 1075 & $1029 \pm 64$ \\
\hline 75 & 234849 & 4556 & 2.19 & 1.2 & 0.2 & 0.467 & 57 & $56 \pm 12$ & 188 & $190 \pm 38$ & 961 & $956 \pm 62$ \\
\hline 76 & 234877 & 4538 & 2.14 & 0.8 & -0.2 & 0.467 & 47 & $52 \pm 10$ & 183 & $175 \pm 34$ & 828 & $849 \pm 63$ \\
\hline 77 & 234898 & 4714 & 2.26 & 0.8 & 0.18 & 0.476 & 44 & $42 \pm 10$ & 167 & $162 \pm 37$ & 984 & $995 \pm 62$ \\
\hline 78 & 234905 & 5042 & 2.28 & 1.3 & -0.12 & 0.478 & 30 & $31 \pm 9$ & 143 & $138 \pm 37$ & 836 & $847 \pm 63$ \\
\hline 79 & 234917 & 5050 & 2.39 & 1.5 & -0.7 & 0.458 & 24 & $16 \pm 12$ & 217 & $234 \pm 38$ & 874 & $859 \pm 62$ \\
\hline 80 & 234926 & 4830 & 2.3 & 1.3 & -0.68 & 0.445 & 36 & $33 \pm 10$ & 140 & $136 \pm 37$ & 934 & $928 \pm 63$ \\
\hline 81 & 234929 & 4893 & 2.35 & 0.8 & -0.68 & 0.483 & 47 & $38 \pm 13$ & 161 & $177 \pm 38$ & 892 & $874 \pm 62$ \\
\hline 82 & 235017 & 4798 & 2.31 & 1.4 & 0 & 0.47 & 47 & $43 \pm 13$ & 156 & $162 \pm 38$ & 1024 & $1012 \pm 62$ \\
\hline 83 & 244618 & 4410 & 2.03 & 1.4 & -0.17 & 0.463 & 42 & $40 \pm 10$ & 161 & $157 \pm 37$ & 899 & $905 \pm 63$ \\
\hline 84 & 244635 & 4650 & 2.24 & 1.5 & -0.19 & 0.435 & 35 & $39 \pm 11$ & 119 & & 855 & \\
\hline 85 & 244642 & 4757 & 2.15 & 1.5 & 0.3 & 0.462 & 24 & $34 \pm 10$ & 185 & $166 \pm 35$ & 830 & $859 \pm 63$ \\
\hline 86 & 244660 & 4736 & 2.17 & 1.3 & -0.28 & 0.43 & 44 & $39 \pm 13$ & 139 & $149 \pm 38$ & 890 & $878 \pm 62$ \\
\hline 87 & 244668 & 4627 & 2.17 & 1.5 & 0.51 & 0.406 & 44 & $62 \pm 11$ & 167 & $119 \pm 36$ & 868 & $880 \pm 63$ \\
\hline 88 & 244704 & 4916 & 2.22 & 1.3 & -0.14 & 0.431 & 31 & $36 \pm 10$ & 156 & $153 \pm 36$ & 989 & $1012 \pm 63$ \\
\hline 89 & 244718 & 4724 & 2.29 & 1.4 & 0.12 & 0.417 & 30 & $31 \pm 9$ & 177 & $171 \pm 37$ & 960 & $974 \pm 63$ \\
\hline 90 & 244727 & 4641 & 2.27 & 1.3 & 0.35 & 0.394 & 36 & $29 \pm 13$ & 107 & $109 \pm 38$ & 823 & $792 \pm 64$ \\
\hline 91 & 244728 & 4491 & 2.03 & 1.5 & 0.3 & 0.463 & 39 & $50 \pm 14$ & 140 & $112 \pm 36$ & 940 & $987 \pm 62$ \\
\hline 92 & 244734 & 4900 & 2.26 & 1.5 & 0.52 & 0.452 & 52 & $44 \pm 11$ & 140 & $147 \pm 39$ & 811 & $754 \pm 64$ \\
\hline 93 & 244742 & 4735 & 2.23 & 1.5 & -0.03 & 0.426 & 53 & $51 \pm 10$ & 158 & $153 \pm 37$ & 952 & $959 \pm 62$ \\
\hline 94 & 244757 & 4910 & 2.23 & 1.2 & -0.53 & 0.419 & 29 & $46 \pm 14$ & 107 & $126 \pm 36$ & 857 & $908 \pm 62$ \\
\hline 95 & 244763 & 4775 & 2.19 & 1.5 & 0.14 & 0.396 & 33 & $33 \pm 10$ & 143 & $136 \pm 37$ & 806 & $822 \pm 63$ \\
\hline 96 & 244817 & 4610 & 2.11 & 1.5 & 0.57 & 0.439 & 40 & $54 \pm 10$ & 149 & $109 \pm 35$ & 866 & $884 \pm 63$ \\
\hline 97 & 244840 & 4605 & 2.11 & 1.5 & 0.45 & 0.412 & 21 & $27 \pm 11$ & 156 & $157 \pm 38$ & 916 & $914 \pm 62$ \\
\hline 98 & 256395 & 4650 & 2.21 & 1.6 & 0.41 & 0.418 & 27 & $27 \pm 13$ & 143 & $142 \pm 38$ & 896 & $892 \pm 62$ \\
\hline 99 & 256401 & 4650 & 2.29 & 1.5 & 0.54 & 0.415 & 47 & $32 \pm 13$ & 28 & $59 \pm 34$ & 931 & $906 \pm 73$ \\
\hline 100 & 256570 & 4843 & 2.19 & 1.5 & 0.56 & 0.42 & 32 & $44 \pm 16$ & 97 & & 681 & \\
\hline 101 & 256579 & 4737 & 2.26 & 1.5 & 0.36 & 0.422 & 17 & $17 \pm 12$ & 115 & $114 \pm 38$ & 699 & $695 \pm 62$ \\
\hline 102 & 256650 & 4598 & 2.19 & 1.5 & 0.44 & 0.412 & 26 & $30 \pm 12$ & 124 & $123 \pm 38$ & 674 & $672 \pm 62$ \\
\hline 103 & 267856 & 4605 & 2.2 & 1.2 & 0.04 & 0.419 & 60 & $61 \pm 11$ & 137 & $141 \pm 37$ & 771 & $769 \pm 62$ \\
\hline 104 & 267862 & 4686 & 2.23 & 1.1 & -0.17 & 0.429 & 23 & $28 \pm 10$ & 127 & $119 \pm 35$ & 747 & $771 \pm 63$ \\
\hline 105 & 267909 & 4716 & 2.16 & 1.4 & 0.43 & 0.416 & 46 & $56 \pm 14$ & 105 & $67 \pm 36$ & 750 & $798 \pm 62$ \\
\hline 106 & 267912 & 4781 & 2.28 & 1.5 & 0.38 & 0.417 & 44 & $36 \pm 11$ & 132 & $138 \pm 38$ & 797 & $746 \pm 64$ \\
\hline 107 & 267985 & 4990 & 2.23 & 1.4 & -0.14 & 0.411 & 16 & $12 \pm 11$ & 85 & $85 \pm 39$ & 660 & $606 \pm 64$ \\
\hline 108 & 268000 & 4568 & 2.2 & 1.5 & 0.19 & 0.416 & 31 & $35 \pm 12$ & 29 & & 740 & \\
\hline 109 & 268011 & 4696 & 2.24 & 1.7 & 0.4 & 0.419 & 36 & $36 \pm 10$ & 126 & $120 \pm 37$ & 751 & $767 \pm 62$ \\
\hline 110 & 268022 & 4968 & 2.26 & 1.5 & 0.22 & 0.385 & 24 & $27 \pm 11$ & 128 & $131 \pm 37$ & 712 & $714 \pm 62$ \\
\hline 111 & 268047 & 4880 & 2.34 & 1.3 & 0.09 & 0.437 & 31 & $28 \pm 10$ & 96 & $99 \pm 38$ & 749 & $691 \pm 64$ \\
\hline 112 & 268068 & 4837 & 2.31 & 1.7 & 0.3 & 0.416 & 39 & $31 \pm 11$ & 144 & $151 \pm 39$ & 767 & $698 \pm 64$ \\
\hline 113 & 385048 & 4686 & 2.24 & 1.2 & -0.36 & 0.486 & 45 & $41 \pm 11$ & 183 & $192 \pm 38$ & 982 & $971 \pm 62$ \\
\hline 114 & 392992 & 4907 & 2.34 & 1.2 & -0.22 & 0.484 & 40 & $44 \pm 10$ & 163 & $156 \pm 34$ & 977 & $998 \pm 63$ \\
\hline 115 & 393009 & 5012 & 2.32 & 1.5 & -0.68 & 0.471 & 33 & $29 \pm 13$ & 176 & $206 \pm 36$ & 1054 & $1093 \pm 63$ \\
\hline 116 & 393012 & 4540 & 2.24 & 1.5 & 0.48 & 0.478 & 64 & $63 \pm 10$ & 181 & $174 \pm 37$ & 1006 & $1028 \pm 62$ \\
\hline 117 & 393024 & 4786 & 2.26 & 1.2 & -1.13 & 0.468 & 35 & $48 \pm 12$ & 112 & $103 \pm 38$ & 1018 & $994 \pm 65$ \\
\hline 118 & 393026 & 4804 & 2.3 & 1.2 & -0.5 & 0.497 & 41 & $42 \pm 10$ & 168 & $171 \pm 35$ & 1039 & $1061 \pm 63$ \\
\hline 119 & 393028 & 4457 & 2.13 & 1.4 & 0.36 & 0.461 & 18 & $14 \pm 10$ & 103 & $109 \pm 39$ & 1011 & $952 \pm 64$ \\
\hline 120 & 393030 & 4574 & 2.18 & 1.5 & 0.35 & 0.475 & 53 & $50 \pm 13$ & 163 & $164 \pm 38$ & 987 & $964 \pm 63$ \\
\hline 121 & 393033 & 4914 & 2.32 & 1 & -0.5 & 0.453 & 43 & $37 \pm 11$ & 213 & $224 \pm 38$ & 976 & $964 \pm 62$ \\
\hline 122 & 393070 & 4903 & 2.3 & 1.6 & 0.47 & 0.459 & 41 & $37 \pm 10$ & 134 & $141 \pm 38$ & 1087 & $1023 \pm 64$ \\
\hline 123 & 393088 & 4575 & 2.21 & 1.5 & 0.22 & 0.466 & 20 & $41 \pm 13$ & 164 & $137 \pm 36$ & 979 & $1007 \pm 63$ \\
\hline 124 & 393091 & 4641 & 2.15 & 1.5 & 0.47 & 0.486 & 34 & $42 \pm 14$ & 142 & & 904 & $944 \pm 62$ \\
\hline 125 & 393111 & 4546 & 2.21 & 1.5 & 0.23 & 0.464 & 31 & $45 \pm 14$ & 157 & $130 \pm 36$ & 1005 & $1048 \pm 62$ \\
\hline 126 & 393117 & 4931 & 2.26 & 0.9 & -0.07 & 0.474 & 36 & $40 \pm 10$ & 173 & $172 \pm 37$ & 1015 & $1033 \pm 63$ \\
\hline 127 & 393124 & 4540 & 2.08 & 1.5 & 0.5 & 0.47 & 17 & $46 \pm 13$ & 192 & $147 \pm 36$ & 1025 & $1050 \pm 63$ \\
\hline
\end{tabular}


Table 3. continued.

\begin{tabular}{|c|c|c|c|c|c|c|c|c|c|c|c|c|}
\hline Star & OgleN & $\begin{array}{c}T_{\mathrm{eff}} \\
\mathrm{K}\end{array}$ & $\begin{array}{c}\log g \\
\text { Dec }\end{array}$ & $\begin{array}{c}\xi \\
\mathrm{km} \mathrm{s}^{-1}\end{array}$ & $\begin{array}{c}{[\mathrm{Fe} / \mathrm{H}]} \\
\mathrm{Dec}\end{array}$ & $E_{B-V^{*}}$ & $\begin{array}{c}\mathrm{EW}(6196) \\
\mathrm{mA}\end{array}$ & $\begin{array}{c}\text { Corr'd } \\
\text { mA }\end{array}$ & $\begin{array}{c}\mathrm{EW}(6204) \\
\mathrm{mA}\end{array}$ & $\begin{array}{c}\text { Corr'd } \\
\mathrm{m} \AA\end{array}$ & $\begin{array}{c}\mathrm{EW}(6284) \\
\mathrm{m} \AA\end{array}$ & $\begin{array}{c}\text { Corr'd } \\
\mathrm{m} \AA\end{array}$ \\
\hline 128 & 393125 & 4528 & 2.09 & 1.4 & 0.29 & 0.488 & 30 & $24 \pm 11$ & 153 & $159 \pm 39$ & 982 & $929 \pm 64$ \\
\hline 129 & 393136 & 4515 & 2.11 & 1.4 & 0.5 & 0.461 & 10 & $19 \pm 14$ & 152 & & 1047 & $1085 \pm 63$ \\
\hline 130 & 393138 & 5073 & 2.34 & 1.4 & -0.64 & 0.473 & 33 & $24 \pm 13$ & 153 & $161 \pm 38$ & 1020 & $999 \pm 64$ \\
\hline 131 & 393152 & 4757 & 2.23 & 1.3 & -0.2 & 0.47 & 39 & $36 \pm 12$ & 189 & $198 \pm 38$ & 1019 & $1009 \pm 62$ \\
\hline 132 & 393162 & 4359 & 2.05 & 1.5 & 0.39 & 0.492 & -15 & $7 \pm 18$ & 161 & & 953 & \\
\hline 133 & 393229 & 4868 & 2.32 & 1.3 & -0.59 & 0.485 & 21 & $29 \pm 13$ & 144 & $138 \pm 34$ & 1021 & $1010 \pm 73$ \\
\hline 134 & 393319 & 4473 & 2.19 & 1.4 & 0.26 & 0.48 & 50 & $40 \pm 12$ & 55 & $58 \pm 38$ & 1045 & $981 \pm 64$ \\
\hline 135 & 393339 & 4972 & 2.41 & 1 & -0.09 & 0.473 & 53 & $45 \pm 12$ & 143 & $148 \pm 38$ & 1007 & $968 \pm 64$ \\
\hline 136 & 402417 & 4655 & 2.2 & 1.2 & -0.2 & 0.486 & 32 & $41 \pm 13$ & 151 & $150 \pm 36$ & 945 & $978 \pm 63$ \\
\hline 137 & 402421 & 4822 & 2.36 & 1.3 & 0.19 & 0.485 & 37 & $41 \pm 11$ & 169 & $174 \pm 38$ & 990 & $990 \pm 62$ \\
\hline 138 & 402432 & 4579 & 2.12 & 1.5 & 0.29 & 0.489 & 19 & $39 \pm 13$ & 187 & $155 \pm 36$ & 951 & $977 \pm 63$ \\
\hline 139 & 402433 & 4808 & 2.24 & 1.3 & -0.19 & 0.461 & 36 & $43 \pm 12$ & 149 & $144 \pm 36$ & 989 & $1010 \pm 63$ \\
\hline 140 & 402435 & 4618 & 2.25 & 1.2 & 0 & 0.482 & 29 & $25 \pm 12$ & 112 & $127 \pm 34$ & 951 & $942 \pm 73$ \\
\hline 141 & 402440 & 4429 & 2.11 & 1.3 & 0.29 & 0.465 & 45 & $43 \pm 13$ & 115 & $116 \pm 38$ & 912 & $904 \pm 62$ \\
\hline 142 & 402449 & 4474 & 2.18 & 1.5 & 0.26 & 0.471 & 23 & $18 \pm 10$ & 125 & $130 \pm 38$ & 1042 & $978 \pm 64$ \\
\hline 143 & 402463 & 5260 & 2.43 & 1.2 & -0.51 & 0.476 & 30 & $31 \pm 10$ & 128 & $131 \pm 35$ & 979 & $1001 \pm 63$ \\
\hline 144 & 402470 & 4270 & 1.97 & 1.2 & 0.16 & 0.457 & 27 & $35 \pm 16$ & 111 & & 902 & \\
\hline 145 & 402477 & 4545 & 2.19 & 1.5 & 0.51 & 0.432 & 37 & $49 \pm 10$ & 196 & $175 \pm 35$ & 827 & $859 \pm 63$ \\
\hline 146 & 402478 & 4983 & 2.35 & 1.2 & -0.53 & 0.47 & 36 & $36 \pm 10$ & 143 & $143 \pm 37$ & 917 & $932 \pm 63$ \\
\hline 147 & 402482 & 4871 & 2.28 & 1.3 & -0.57 & 0.478 & 34 & $30 \pm 10$ & 118 & $120 \pm 39$ & 910 & $875 \pm 64$ \\
\hline 148 & 402502 & 4818 & 2.19 & 1.2 & -0.95 & 0.476 & 32 & $32 \pm 9$ & 146 & $146 \pm 37$ & 840 & $846 \pm 63$ \\
\hline 149 & 402516 & 4936 & 2.2 & 1.3 & -0.65 & 0.46 & 27 & $22 \pm 10$ & 130 & $133 \pm 39$ & 791 & $761 \pm 64$ \\
\hline 150 & 402518 & 5034 & 2.32 & 1 & -0.87 & 0.446 & 39 & $37 \pm 9$ & 133 & $133 \pm 37$ & 858 & $865 \pm 63$ \\
\hline 151 & 402550 & 4420 & 2.15 & 1.5 & 0.39 & 0.475 & 46 & $38 \pm 11$ & 69 & $76 \pm 39$ & 845 & $772 \pm 64$ \\
\hline 152 & 402560 & 4984 & 2.37 & 1.1 & -1.01 & 0.441 & 35 & $35 \pm 9$ & 146 & $145 \pm 37$ & 825 & $826 \pm 63$ \\
\hline 153 & 402600 & 5158 & 2.4 & 1.3 & -0.52 & 0.421 & 32 & $25 \pm 12$ & 132 & $146 \pm 38$ & 823 & $810 \pm 62$ \\
\hline 154 & 402621 & 4913 & 2.28 & 1.3 & -0.56 & 0.45 & 37 & $35 \pm 13$ & 142 & $164 \pm 36$ & 767 & $804 \pm 63$ \\
\hline 155 & 402632 & 4937 & 2.32 & 1.5 & 0.55 & 0.439 & 34 & $30 \pm 10$ & 130 & $137 \pm 39$ & 635 & $572 \pm 64$ \\
\hline 156 & 402641 & 4775 & 2.18 & 1.5 & 0.49 & 0.458 & 23 & $36 \pm 10$ & 120 & $90 \pm 34$ & 782 & $803 \pm 63$ \\
\hline 157 & 412854 & 5191 & 2.4 & 1.4 & -0.69 & 0.419 & 34 & $31 \pm 10$ & 105 & $107 \pm 39$ & 658 & $627 \pm 64$ \\
\hline 158 & 412889 & 4717 & 2.23 & 1.6 & 0.42 & 0.4 & 39 & $34 \pm 13$ & 108 & $108 \pm 38$ & 665 & $635 \pm 64$ \\
\hline 159 & 412894 & 5118 & 2.31 & 1.5 & -0.45 & 0.427 & 25 & $30 \pm 12$ & 99 & $97 \pm 34$ & 724 & $715 \pm 73$ \\
\hline 160 & 412898 & 4871 & 2.22 & 1.8 & 0.07 & 0.415 & 22 & $18 \pm 10$ & 126 & $130 \pm 38$ & 627 & $570 \pm 64$ \\
\hline 161 & 412921 & 4617 & 2.13 & 1.5 & 0.47 & 0.413 & 69 & $79 \pm 14$ & 99 & $60 \pm 36$ & 601 & $647 \pm 62$ \\
\hline 162 & 412957 & 4920 & 2.23 & 1.5 & -0.54 & 0.422 & 21 & $19 \pm 10$ & 105 & $105 \pm 39$ & 604 & $568 \pm 64$ \\
\hline 163 & 412979 & 4756 & 2.24 & 1.5 & -0.58 & 0.424 & 36 & $32 \pm 10$ & 134 & $133 \pm 37$ & 652 & $643 \pm 62$ \\
\hline 164 & 413012 & 4869 & 2.29 & 1.2 & -0.15 & 0.408 & 32 & $36 \pm 10$ & 144 & $143 \pm 37$ & 685 & $704 \pm 63$ \\
\hline 165 & 413043 & 4974 & 2.24 & 1.5 & 0.09 & 0.446 & 34 & $35 \pm 10$ & 83 & & 699 & \\
\hline 166 & 413060 & 4811 & 2.29 & 1.1 & -0.37 & 0.422 & 29 & $31 \pm 10$ & 136 & $136 \pm 37$ & 724 & $741 \pm 63$ \\
\hline 167 & 413066 & 4674 & 2.11 & 1.5 & 0.44 & 0.419 & 33 & $33 \pm 10$ & 193 & $186 \pm 37$ & 684 & $709 \pm 63$ \\
\hline 168 & 423393 & 4702 & 2.28 & 1.5 & 0.41 & 0.408 & 24 & $25 \pm 10$ & 150 & $143 \pm 37$ & 696 & $718 \pm 63$ \\
\hline 169 & 423398 & 4351 & 2.05 & 1.3 & 0.42 & 0.426 & 35 & $47 \pm 10$ & 182 & $156 \pm 34$ & 646 & $672 \pm 63$ \\
\hline 170 & 423427 & 4818 & 2.22 & 1.5 & 0.33 & 0.479 & 41 & $30 \pm 12$ & 126 & $132 \pm 38$ & 688 & $646 \pm 64$ \\
\hline 171 & 423432 & 4945 & 2.36 & 1.4 & -0.55 & 0.409 & 36 & $44 \pm 14$ & 119 & $143 \pm 36$ & 727 & $773 \pm 62$ \\
\hline 172 & 423434 & 4753 & 2.31 & 1.5 & -0.15 & 0.502 & 28 & $25 \pm 12$ & 145 & $153 \pm 38$ & 759 & $749 \pm 62$ \\
\hline 173 & 423461 & 4743 & 2.24 & 1 & -0.13 & 0.48 & 27 & $24 \pm 12$ & 165 & $173 \pm 38$ & 668 & $659 \pm 62$ \\
\hline 174 & 423485 & 4779 & 2.3 & 1.5 & 0.1 & 0.421 & 11 & $23 \pm 14$ & 108 & $94 \pm 36$ & 692 & $741 \pm 62$ \\
\hline 175 & 423486 & 5448 & 2.42 & 1.3 & -0.39 & 0.483 & 28 & $32 \pm 13$ & 112 & $121 \pm 36$ & 695 & $723 \pm 63$ \\
\hline 176 & 423511 & 4472 & 2.03 & 1.2 & -0.11 & 0.458 & 31 & $36 \pm 10$ & 153 & $147 \pm 36$ & 780 & $805 \pm 63$ \\
\hline 177 & 423516 & 4808 & 2.18 & 1.2 & 0.4 & 0.472 & 4 & $29 \pm 13$ & 124 & $85 \pm 36$ & 727 & $754 \pm 63$ \\
\hline 178 & 423530 & 4922 & 2.28 & 1 & -0.11 & 0.415 & 51 & $49 \pm 10$ & 152 & $148 \pm 37$ & 807 & $811 \pm 62$ \\
\hline 179 & 423539 & 4676 & 2.11 & 1.5 & 0.54 & 0.418 & 36 & $50 \pm 10$ & 144 & $104 \pm 35$ & 774 & $791 \pm 63$ \\
\hline 180 & 423541 & 4491 & 2.18 & 1.4 & 0.2 & 0.447 & 39 & $34 \pm 10$ & 116 & $120 \pm 39$ & 710 & $649 \pm 64$ \\
\hline 181 & 423543 & 4975 & 2.4 & 1.4 & -0.91 & 0.433 & 32 & $34 \pm 10$ & 87 & $80 \pm 39$ & 684 & $659 \pm 64$ \\
\hline 182 & 423551 & 4911 & 2.28 & 1.5 & 0.14 & 0.42 & 24 & $19 \pm 10$ & 136 & $141 \pm 38$ & 869 & $811 \pm 64$ \\
\hline 183 & 423569 & 4598 & 2.18 & 1.5 & 0.45 & 0.442 & 42 & $52 \pm 10$ & 183 & $180 \pm 37$ & 688 & $711 \pm 63$ \\
\hline 184 & 423573 & 5164 & 2.39 & 1.1 & -0.59 & 0.421 & 25 & $24 \pm 10$ & 119 & $120 \pm 37$ & 764 & $780 \pm 63$ \\
\hline 185 & 423604 & 4422 & 2.07 & 1.3 & 0.55 & 0.422 & 21 & $21 \pm 13$ & 96 & $93 \pm 38$ & 736 & $731 \pm 62$ \\
\hline 186 & 423876 & 4671 & 2.29 & 1.5 & 0.02 & 0.469 & 36 & $42 \pm 12$ & 105 & & 618 & \\
\hline 187 & 545299 & 4483 & 2.16 & 1 & 0.17 & 0.486 & 52 & $48 \pm 13$ & 144 & $147 \pm 38$ & 1111 & $1093 \pm 62$ \\
\hline 188 & 545326 & 4578 & 2.16 & 1.1 & 0.2 & 0.524 & 8 & $20 \pm 14$ & 102 & & 1260 & $1307 \pm 63$ \\
\hline 189 & 545421 & 5012 & 2.39 & 1.2 & -0.22 & 0.492 & 45 & $49 \pm 10$ & 170 & $162 \pm 34$ & 1047 & $1068 \pm 63$ \\
\hline 190 & 545444 & 4407 & 2.07 & 1.5 & 0.48 & 0.485 & 23 & $15 \pm 11$ & 63 & $69 \pm 39$ & 991 & $936 \pm 64$ \\
\hline
\end{tabular}


H.-C. Chen et al.: Extracting interstellar diffuse absorption bands from cool star spectra

Table 3. continued.

\begin{tabular}{|c|c|c|c|c|c|c|c|c|c|c|c|c|}
\hline Star & OgleN & $\begin{array}{c}T_{\text {eff }} \\
\mathrm{K}\end{array}$ & $\begin{array}{c}\log g \\
\text { Dec }\end{array}$ & $\begin{array}{c}\xi \\
\mathrm{km} \mathrm{s}^{-1}\end{array}$ & $\begin{array}{c}{[\mathrm{Fe} / \mathrm{H}]} \\
\mathrm{Dec}\end{array}$ & $E_{B-V^{*}} *$ & $\begin{array}{c}\mathrm{EW}(6196) \\
\mathrm{m} \AA\end{array}$ & $\begin{array}{c}\text { Corr'd } \\
\text { mA }\end{array}$ & $\begin{array}{c}\mathrm{EW}(6204) \\
\mathrm{m} \AA\end{array}$ & $\begin{array}{c}\text { Corr'd } \\
\mathrm{m} \AA\end{array}$ & $\begin{array}{c}\mathrm{EW}(6284) \\
\mathrm{mA}\end{array}$ & $\begin{array}{c}\text { Corr'd } \\
\mathrm{m \AA}\end{array}$ \\
\hline 191 & 545498 & 4806 & 2.26 & 1.5 & -0.5 & 0.484 & 31 & $31 \pm 10$ & 159 & $160 \pm 37$ & 995 & $1012 \pm 63$ \\
\hline 192 & 545519 & 4917 & 2.41 & 1.5 & -0.31 & 0.483 & 58 & $60 \pm 10$ & 169 & & 1173 & \\
\hline 193 & 545749 & 4556 & 2.25 & 1.4 & 0.04 & 0.485 & 44 & $42 \pm 12$ & 155 & $160 \pm 38$ & 1003 & $995 \pm 62$ \\
\hline 194 & 554729 & 4752 & 2.28 & 1.2 & -0.83 & 0.474 & 41 & $38 \pm 10$ & 162 & $190 \pm 36$ & 997 & $1025 \pm 63$ \\
\hline 195 & 554738 & 4701 & 2.32 & 1.2 & 0.13 & 0.511 & 40 & $38 \pm 10$ & 148 & $142 \pm 37$ & 1007 & $1020 \pm 62$ \\
\hline 196 & 554754 & 5056 & 2.3 & 1.4 & -0.52 & 0.485 & 41 & $35 \pm 11$ & 191 & $201 \pm 38$ & 949 & $937 \pm 62$ \\
\hline 197 & 554762 & 4625 & 2.29 & 1.4 & 0.13 & 0.484 & 44 & $45 \pm 11$ & 155 & $157 \pm 37$ & 959 & $960 \pm 62$ \\
\hline 198 & 554807 & 4941 & 2.4 & 1.4 & -0.04 & 0.484 & 32 & $28 \pm 13$ & 92 & $104 \pm 34$ & 887 & $867 \pm 73$ \\
\hline 199 & 554820 & 4824 & 2.37 & 1.5 & 0.24 & 0.483 & 33 & $22 \pm 12$ & 84 & $99 \pm 34$ & 815 & \\
\hline 200 & 554835 & 4622 & 2.17 & 1.3 & 0.48 & 0.455 & 13 & $30 \pm 17$ & 105 & & 882 & \\
\hline 201 & 554849 & 4581 & 2.19 & 1.5 & 0.46 & 0.47 & 43 & $61 \pm 12$ & 225 & $180 \pm 36$ & 861 & $874 \pm 63$ \\
\hline 202 & 554860 & 4877 & 2.24 & 1.3 & -0.32 & 0.477 & 38 & $32 \pm 13$ & 147 & $157 \pm 38$ & 814 & $802 \pm 62$ \\
\hline 203 & 554876 & 4949 & 2.38 & 1.5 & -0.03 & 0.447 & 29 & $24 \pm 10$ & 131 & $135 \pm 38$ & 748 & $696 \pm 64$ \\
\hline 204 & 554901 & 4638 & 2.11 & 1.2 & -0.67 & 0.45 & 31 & $34 \pm 12$ & 99 & $93 \pm 38$ & 714 & $677 \pm 64$ \\
\hline 205 & 564834 & 5356 & 2.41 & 1.3 & -0.36 & 0.424 & 34 & $32 \pm 10$ & 134 & $130 \pm 37$ & 772 & $773 \pm 63$ \\
\hline 206 & 564840 & 4713 & 2.23 & 1.5 & 0.33 & 0.435 & 23 & $8 \pm 12$ & 37 & $54 \pm 34$ & 734 & \\
\hline 207 & 564857 & 4912 & 2.28 & 1.4 & 0.01 & 0.448 & 21 & $21 \pm 10$ & 122 & $116 \pm 37$ & 695 & $708 \pm 63$ \\
\hline 208 & 564949 & 4619 & 2.14 & 1.5 & 0.4 & 0.433 & 23 & $45 \pm 13$ & 192 & $153 \pm 36$ & 765 & $787 \pm 63$ \\
\hline 209 & 564992 & 5269 & 2.37 & 1.5 & -0.61 & 0.432 & 30 & $29 \pm 10$ & 142 & $143 \pm 37$ & 685 & $700 \pm 63$ \\
\hline 210 & 565019 & 4801 & 2.29 & 1.5 & 0.38 & 0.427 & 46 & $36 \pm 12$ & 121 & $127 \pm 38$ & 656 & $611 \pm 64$ \\
\hline 211 & 565025 & 4729 & 2.19 & 1.4 & -0.58 & 0.426 & 32 & $29 \pm 10$ & 118 & $119 \pm 38$ & 629 & $594 \pm 64$ \\
\hline 212 & 565056 & 5067 & 2.39 & 1.6 & 0.63 & 0.43 & 60 & $68 \pm 14$ & 56 & $2 \pm 37$ & 717 & $766 \pm 62$ \\
\hline 213 & 565094 & 5049 & 2.32 & 1.5 & 0.14 & 0.515 & 26 & $20 \pm 13$ & 119 & $131 \pm 35$ & 718 & $687 \pm 71$ \\
\hline 214 & 575390 & 4639 & 2.1 & 1.5 & 0.18 & 0.494 & 40 & $47 \pm 10$ & 147 & $144 \pm 37$ & 692 & $714 \pm 63$ \\
\hline 215 & 575421 & 4685 & 2.19 & 1.2 & -0.08 & 0.477 & 31 & $30 \pm 10$ & 162 & $157 \pm 37$ & 658 & $669 \pm 63$ \\
\hline 216 & 575448 & 4583 & 2.19 & 1.3 & 0.58 & 0.467 & 39 & $27 \pm 13$ & 25 & $42 \pm 36$ & 675 & $631 \pm 69$ \\
\hline 217 & 575485 & 4634 & 2.28 & 1.1 & -0.09 & 0.483 & 32 & $35 \pm 9$ & 189 & $186 \pm 37$ & 707 & $721 \pm 63$ \\
\hline 218 & 575542 & 4636 & 2.21 & 1.5 & 0.58 & 0.425 & 24 & $24 \pm 13$ & 124 & $120 \pm 38$ & 703 & $699 \pm 62$ \\
\hline 219 & 575585 & 4769 & 2.19 & 1.2 & -0.17 & 0.441 & 35 & $27 \pm 12$ & 104 & $109 \pm 38$ & 671 & $636 \pm 64$ \\
\hline
\end{tabular}

